\title{
Sgol2 provides a regulatory platform that coordinates essential cell cycle processes during meiosis I in oocytes
}

\section{Ahmed Rattani ${ }^{1}$, Magda Wolna ${ }^{1 \dagger}$, Mickael Ploquin ${ }^{1 \dagger \neq a}$, Wolfgang Helmhart ${ }^{1 \dagger \neq b}$, Seamus Morrone ${ }^{2}$, Bernd Mayer ${ }^{3}$, Jonathan Godwin ${ }^{1}$, Wenqing $\mathrm{Xu}^{2}$, Olaf Stemmann ${ }^{3}$, Alberto Pendas ${ }^{4}$, Kim Nasmyth ${ }^{1 *}$}

${ }^{1}$ Department of Biochemistry, University of Oxford, Oxford, United Kingdom; ${ }^{2}$ Department of Biological Structure, University of Washington, Seattle, United States; ${ }^{3}$ Department of Genetics, University of Bayreuth, Bayreuth, Germany; ${ }^{4}$ Campus Miguel de Unamúno, Instituto de Biología Molecular y Celular del Cáncer (CSIC-USAL), Salamanca, Spain

Abstract Accurate chromosome segregation depends on coordination between cohesion resolution and kinetochore-microtubule interactions (K-fibers), a process regulated by the spindle assembly checkpoint (SAC). How these diverse processes are coordinated remains unclear. We show that in mammalian oocytes Shugoshin-like protein 2 (Sgol2) in addition to protecting cohesin, plays an important role in turning off the SAC, in promoting the congression and bi-orientation of bivalents on meiosis I spindles, in facilitating formation of K-fibers and in limiting bivalent stretching. Sgol2's ability to protect cohesin depends on its interaction with PP2A, as is its ability to silence the SAC, with the latter being mediated by direct binding to Mad2. In contrast, its effect on bivalent stretching and K-fiber formation is independent of PP2A and mediated by recruitment of MCAK and inhibition of Aurora $C$ kinase activity respectively. By virtue of its multiple interactions, Sgol2 links many of the processes essential for faithful chromosome segregation.

DOI: 10.7554/eLife.01133.001

\section{Introduction}

The production of haploid gametes from diploid germ cells depends on two rounds of chromosome segregation (meiosis I and II) without an intervening round of DNA replication. Defects during the first or second meiotic division in oocytes lead to formation of aneuploid eggs, which in humans occurs with a frequency between 10 and $30 \%$ and is a major cause of fetal miscarriages (Hassold and Hunt, 2001). Understanding the causes of meiotic chromosome missegregation will require clarifying not only the forces and regulatory mechanisms governing meiotic chromosome segregation but also how these are coordinated.

At the heart of this process are two opposing forces. The pulling forces produced by kinetochoremicrotubules attachments (K-fibers) and resisting forces generated by sister chromatid cohesion, which counteracts K-fiber forces if and when kinetochores attach to microtubules with different polarities. During meiosis I, cohesion along chromosome arms holds bivalent chromosomes together following the creation of chiasmata produced by reciprocal recombination between homologous non-sister chromatids. This cohesion must persist during the attachment of maternal and paternal kinetochores to microtubules from opposite poles (bi-orientation) and resist the resulting spindle forces. The degree of traction exerted by meiotic spindles must create sufficient tension to facilitate bi-orientation (Tachibana-Konwalski et al., 2010). During the bi-orientation process, the initial attachment of kinetochores to the surface of the microtubule lattice (lateral attachment) is converted to attachments 
eLife digest Human reproductive cells-eggs and sperm—are produced through a process called meiosis. This means a 'parent' cell in the ovaries or testes undergoes two stages of cell division: it first divides into two cells, which then divide again to produce four 'daughter' cells. A crucial part of meiosis is ensuring that each daughter cell has half the number of chromosomes that the parent cell did.

Before the first round of meiosis, the chromosomes in the parent cell are copied to produce enough chromosomes for the four daughter cells. The distribution of these chromosomes between the daughter cells is determined by the opposing forces acting on them. The pairs of identical chromosomes produced during the copying process are held together by a proteinaceous glue, while microtubules attached to a structure called the spindle-which has poles at opposite ends of the cell-try to pull these pairs of chromosomes apart.

Rattani et al. now show that a protein called shugoshin-like 2 (Sgol2), which is involved in holding pairs of identical chromosomes together after the first round of division, has a bigger role than was previously realised. Sgol2 performs three other functions: it helps to align the chromosomes prior to division by, it is thought, facilitating the formation of the K-fibers that attach the microtubules to the chromosomes; it turns off the checkpoint that monitors the alignment of the chromosomes and the attachment of the microtubules; and it regulates a number of the enzymes involved in the process. The specific interactions that allow Sgol2 to perform these diverse functions in meiosis were also identified. Thus, Rattani et al. show that in linking so many essential processes throughout meiosis, Sgol2 appears to have a key, if not unique, role in determining the fate of chromosomes as cells divide.

DOI: 10.7554/eLife.01133.002

to their plus ends (end on attachment), creating K-fibers. Because this process is intrinsically error prone, inappropriate attachments, for example those that connect maternal and paternal kinetochores to the same pole, must be disrupted through phosphorylation of kinetochore proteins by Aurora B/C kinases (Kitajima et al., 2011). However, because these kinases disrupt K-fibers, they must subsequently be down regulated once bivalents bi-orient correctly.

The first meiotic division is eventually triggered by activation of a gigantic ubiquitin protein ligase called the anaphase-promoting complex or cyclosome (APC/C) whose destruction of securin and cyclin B activates a thiol protease called separase that cleaves the kleisin subunit of the cohesin complex holding sister chromatids together (McGuinness et al., 2005; Kudo et al., 2006). This process converts chromosomes from bivalents to dyads. It is delayed until all bivalents have bi-oriented by the production, at kinetochores that have not yet come under tension, of a potent inhibitor of the APC/C called the mitotic checkpoint complex (MCC) whose Mad2 subunit binds tightly to the APC/C's Cdc20 co-activator protein. This regulatory mechanism, called the spindle assembly checkpoint (SAC), must be turned off before $\mathrm{APC} / \mathrm{C}^{\mathrm{Cdc} 20}$ can direct destruction of securin and cyclin $\mathrm{B}$ and thereby activate separase. Another pre-condition for cleavage, at least in yeast, is phosphorylation of cohesin's kleisin

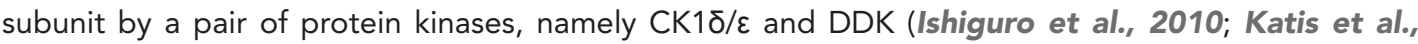
2010). During the first meiotic division, cohesin is phosphorylated along chromosome arms but not at centromeres, which ensures that only cohesion along arms is destroyed by separase at the onset of anaphase I. The consequent persistence of cohesion at centromeres promotes bi-orientation of dyads during meiosis II.

Centromeric cohesin avoids phosphorylation and therefore cleavage during the first meiotic division because separase activation is preceded by the recruitment to centromeres of orthologues of the Drosophila MEI-S332 protein, called shugoshins. Members of this family contain a conserved C-terminal basic region and an $\mathrm{N}$-terminal homodimeric parallel coiled coil, which provides a docking site for protein phosphatase 2A's (PP2A) C and B' subunits (Xu et alo, 2009). In budding yeast, mutant proteins defective specifically in PP2A binding fail to confer protection of centromeric cohesion during meiosis I (Xu et al., 2009).

Mammals have two members of the shugoshin family: Shugoshin-like protein 1 (Sgol1), and Shugoshin-like protein 2 (Sgol2). The former prevents centromeric cohesin from a process called the 'prophase pathway' that removes cohesin from chromosomes by a non-proteolytic mechanism soon 
after cells enter mitosis (McGuinness et al., 2005; Liu et al., 2013). Sgol2, on the other hand, protects centromeric cohesin from separase at the first meiotic division (Lee et al., 2008; Llano et al., 2008). Sgol2's coiled coil domain binds PP2A in vitro (Xu et al., 2009) but whether this is vital for protecting centromeric cohesion is not known. Sgol2 also interacts with MCAK (Huang et alo, 2007), a microtubule depolymerizing kinesin, implicated in correcting inappropriate kinetochore-microtubule interactions (error correction), and with Mad2 an essential component of the MCC (Orth et al., 2011). Shugoshins, though not explicitly Sgol2, have also been implicated in recruiting to kinetochores the Aurora B kinase, necessary both for the SAC and for error correction (Tsukahara et al., 2010; Yamagishi et al., 2010). Based on its interactions, Sgol2 has been linked to cohesion protection, the spindle assembly checkpoint, and error correction pathways. However, the physiological significance of these multiple interactions remain unclear.

We show here that Sgol2 defective in PP2A binding fails to protect centromeric cohesin, as found for Sgo1 in yeast (Xu et al., 2009). However, if this were the sole function of Sgol2, then chromosome behavior during meiosis I should be unaffected. To our surprise, we found that Sgol2 deficiency caused striking changes in chromosome and microtubule dynamics. It delayed bi-orientation of bivalents on meiosis I spindles, caused increased bivalent stretching, and greatly increased Aurora B/C kinase activity at kinetochores, which was accompanied by an increase in lateral and a decrease in end on kinetochore-microtubules attachments. Lastly, Sgol2 deficiency delayed considerably APC/C Cdc20 activation, suggesting that it was required to shut off the SAC. Sgol2 helps to turn off the SAC by binding directly both PP2A and the MCC protein Mad2, it moderates chromosome stretching by recruiting to kinetochores the kinesin MCAK, and likely promotes formation of K-fibers by down-regulating the activity of Aurora B/C kinase specifically at kinetochores. Meiotic chromosome segregation is a highly complex process dependent on an array of different biochemical processes. Our findings imply that through its multi-domain structure, Sgol2 has an important if not unique role in coordinating many of the key processes within this array.

\section{Results}

\section{Sgol2 interacts with PP2A through conserved residues in its coiled coil}

An essential feature of shugoshins is a conserved $\mathrm{N}$-terminal homodimeric coiled coil that binds PP2A (Figure 1A). Unlike Sgol1, which only interacts stably with the PP2A holoenzyme (a complex of A, B' and $C$ subunits), Sgol 2 also associates with a core complex containing only $A$ and $C$ subunits ( $X u$ et al., 2009). To create versions of Sgol 2 defective specifically in binding PP2A, conserved residues (namely L51, N54, R56, and E82) predicted to interact directly with PP2A were substituted by alanine or in the case of E82 also by lysine (Figure 1B). Likewise, the highly conserved asparagine (N55 on Sgol2), corresponding to N61 in Sgol1, which forms a hydrogen bond within the coiled coil enabling it to adopt a conformation compatible with PP2A binding, was substituted by isoleucine (Figure 1B). Importantly, binding between purified Flag- and His-tagged Sgol2 fragments spanning the coiled coil (cc) region (31-117) was unaffected by any of these mutations, implying that all permit coiled coil formation. A triple mutation L51A/N54A/R56A (3A) reduced dimerization modestly (Figure 1D) but this effect was not seen when the two proteins were co-expressed in bacteria (Figure 1E). To assess the effects on PP2A binding, GST-tagged PP2A A subunit was incubated with $\mathrm{B}^{\prime}$ and $C$ subunits in the presence of MBP-Sgol2cc-Flag. Pull down experiments revealed that N55I and the 3A triple mutation abolished co-purification of Sgol2 with the GST-tagged A subunit while N54A and R56A reduced it. In contrast, L51A and E82A had little or no effect while E82K caused a modest reduction (Figure 1F).

\section{Sgol2 protects centromeric cohesion by recruiting PP2A to kinetochores}

To investigate whether association of PP2A with Sgol2's coiled coil is necessary for protecting centromeric cohesion, we compared the effect of the mutations on PP2A's co-localization with Sgol2 at kinetochores with their effect on the maintenance of cohesion after oocytes have undergone the first meiotic division. Sgol2 and PP2A co-localize at inner kinetochores of bivalent chromosomes at metaphase I in wild type oocytes (Figure 2A) but neither protein was detected at this location in sgol2 ${ }^{\Delta / \Delta}$ oocytes $(n=26)$. Importantly, microinjection of wild type Sgol 2 encoding mRNA into sgol2 $2^{\Delta / \Delta}$ oocytes restored accumulation at kinetochores of both Sgol2 and PP2A at metaphase I. In contrast, microinjection of mRNAs carrying N54A, N55I, or 3A mutations restored Sgol2 but not PP2A (Figure 2A), implying 
A

\section{B}

Sgol1 H.s.(51-99) STLLKNYQDNNKMLVLALENEKSKVKEAQD I I LQLRKECYYLTCQLYAL Sgol1 E.c.(51-99) STLLKNYQDNNRML VLALENEKSKVREAED I I LQLRKECYYLTCQLYTL Sgol1 C.f.(51-99) ST LL LNYQDNNRML VLALENEKSKVREAQD I I LQLRRECYYLTCQLYAL Sgol1 R.n.(51-99) ST LLKYYQDNNRL LVLALENEKSKVREAQD I I LQLRRECYHLTCQLCTL Sgol1 M.m.(51-99)AT LLRYYQDNNRL LVLALENEKSKVREAQDV I LQLRKECYYLTCQLYAL Sgol2 H.s.(48-96) SI FK I SL KHNNRAL AQAL SREKENSRR I T TEKML LQKEVEKLNFENTFL Sgol2 E.c.(48-96) S I FK I SL KHNNRAL AQALSREKENSRR I T TEKML LQKEVEKLNFENTF L Sgol2 C.f.(48-96) S I FK I SLKHNNRAL AQAL SREKENYRR I T TEKML LQKEVEKLNFENAFL Sgol2 R.n.(45-93)S I FK I SLKHNNRALARALSKEKENSRR I T TEKVQLQKEVEKLNFENTF L Sgol2 M.m.(45-93) S I FK I SLKHNNRALARALSKEKENSRR I T TEKMQLQKEVEKLNFENTFL

D

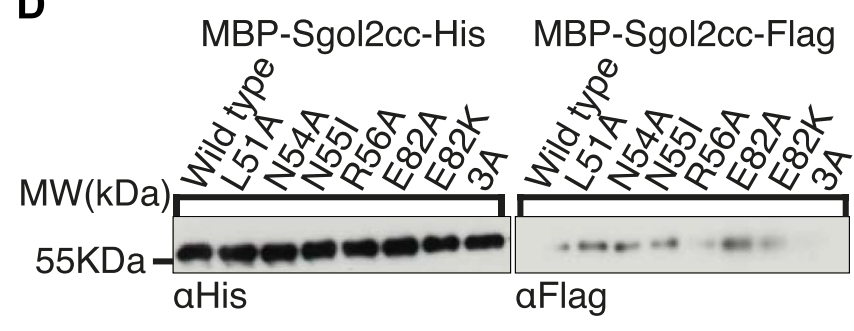

MBP-Sgol2cc-His MBP-Sgol2cc-Flag
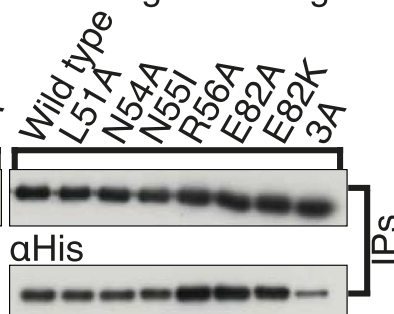

aFlag
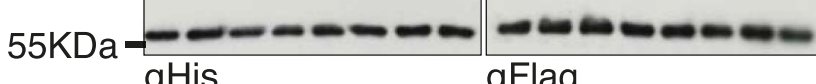

aFlag

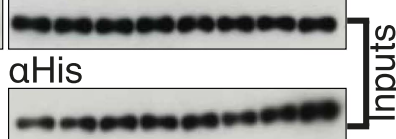

aFlag

$\mathbf{F}$

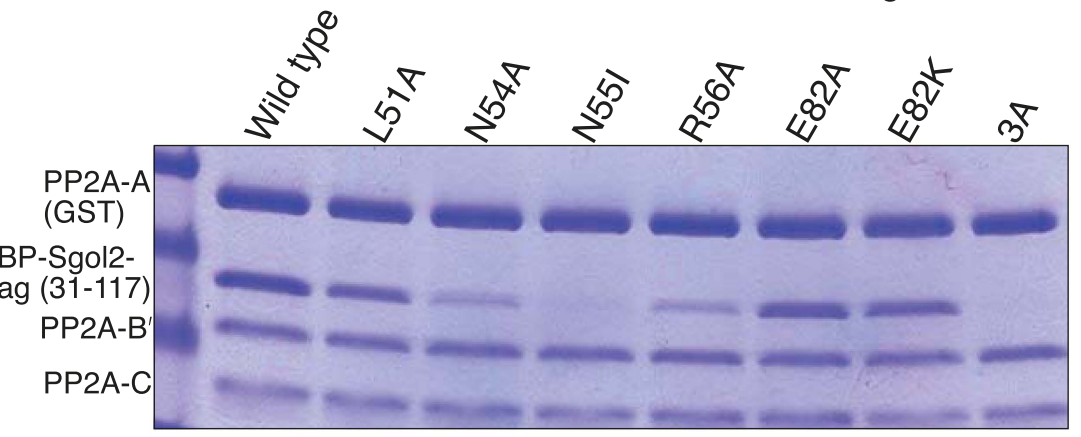

C

Madl1 H.s.(537-552)Q S R T K V L H M S - L N P T S V Madl1 M.m.(537-552)Q S R T K V L H M S - L N P I SM Cdc20 H.s.(125-140) V E E A K I L R L S - G K P Q N A Cdc20 M.m.(125-140)V E E A K I LR L S - G K P Q N A Sgol1 X.I.(163-178) S G A S A ILR L P S H A P IS Sgol2 H.s.(146-161) S K Q C K LM R L P F A R - V P L Sgol2 M.m.(143-158) S K Q C K P A H L P Y A R - V L L MIM

\section{E}

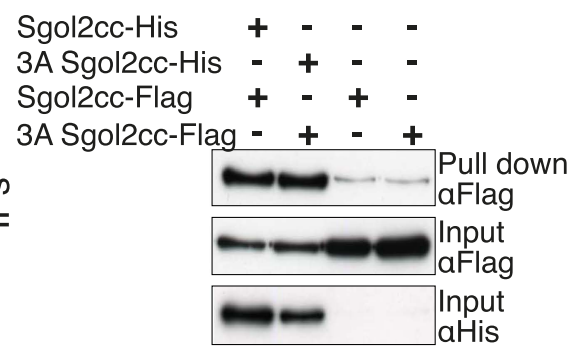

G M.m. Sgol2 $(120-180)$

X.ISgol1

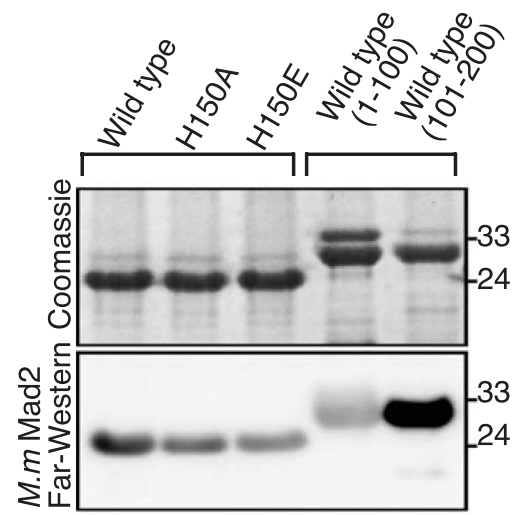

Figure 1. Sgol2 binds to PP2A and Mad2 through coiled coil domain and Mad2 Interaction Motif (MIM), respectively. (A) Schematic diagram indicating relative positions of the coiled coil domain and the MIM sequence in M. musculus Sgol2 (not drawn to scale). (B) Sequence alignment of the coiled coil domains of Sgol1 and Sgol2 from different vertebrate species. Arrowheads below the alignment indicate conserved residues (L51, N54, R56, and E82) in the coiled coil domain of Sgol2 mapping onto binding sites between PP2A and Sgol1. The conserved asparagine residue (N55 in mSgol2), important for maintaining the interaction surface of the coiled coil domain, is marked by an arrowhead above the alignment. (C) Sequence alignment of conserved MIM (Mad2 Interaction Motif) in Sgol2, Mad1, and Cdc20 from Xenopus, human and mouse origin. (D) Dimerization assay of coiled coil domains from wild type and mutant Sgol2. The Flag-tagged MBP-Sgol2cc (31-117) was pulled down by the His-tagged MBP-Sgol2cc (31-117) with the same substitution. Homodimerisation was assessed by the relative abundance of each form in the IP by western blots. (E) Co-immunoprecipitation assay of MBP-Sgol2cc from wild type and 3A mutant are shown to validate the homodimerisation of coiled coil domains. MBP-Sgol2cc-Flag and MBP-Sgol2cc-His were expressed either independently, or co-expressed. After His-tag pull-down, each fraction was separated on a 12\% SDS-PAGE and analyzed by immunoblotting with anti-Flag and anti-His antibodies. Input was resolved on a 12\% SDS-PAGE. (F) The in vitro binding assay of PP2A A(GST-tagged), B', and $\mathrm{C}$ subunits with wild type MBP-mSgol2cc-Flag and variants thereof. After GST pull down, eluates were resolved by SDS-PAGE and Coomassie stained. (G) Sgol2 and Mad2 binding assay. Sgol2 fragment spanning amino-acid (aa) residues 120-180 with wild type and mutant MIM sequences were assessed for Mad2 interaction by far-western blot. Fragments from Xenopus Sgol1, with and without MIM domain, were used as positive and negative controls. DOI: 10.7554/eLife.01133.003 
A
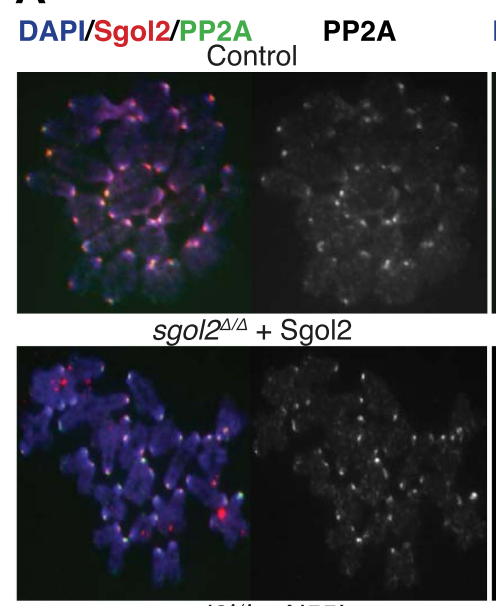

DAPI/Sgol2/PP2A

PP2A
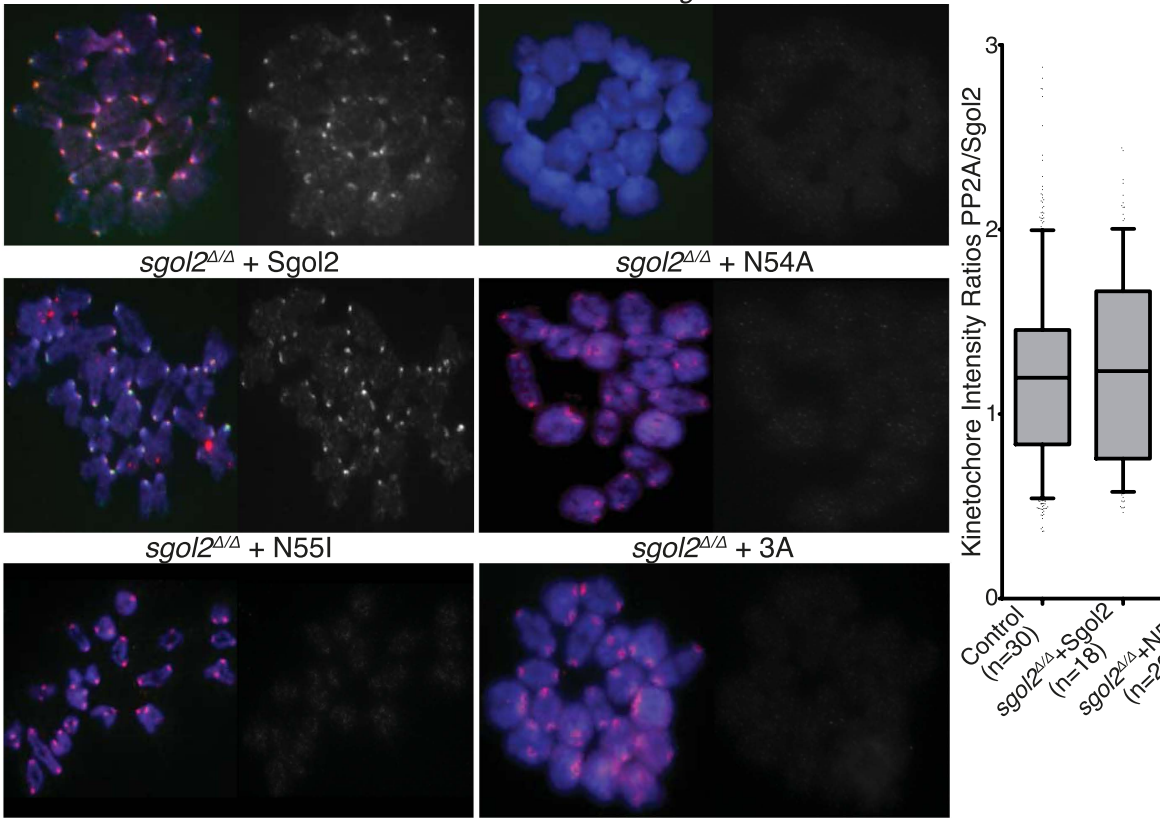

B

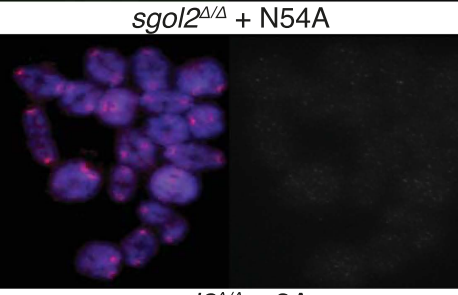

$g o l 2^{\Delta / \Delta}+3 A$
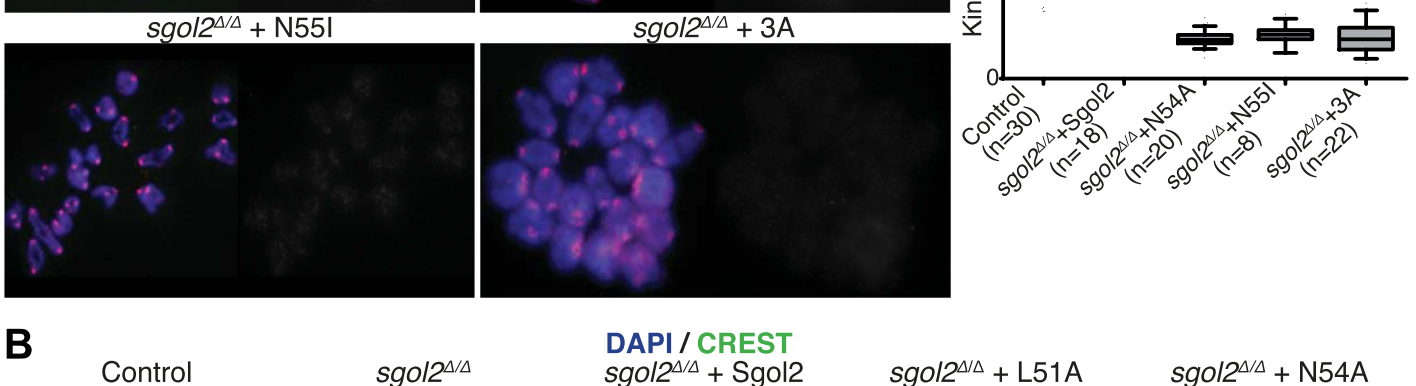

$\operatorname{sgol}^{\Delta / \Delta}$

DAPI / CREST

$\mathrm{sgol}^{\Delta / \Delta}+\mathrm{Sgol} 2$

sgol2 $^{\Delta / \Delta}+\mathrm{L} 51 \mathrm{~A}$

$s g o l 2^{\Delta / \Delta}+\mathrm{N} 54 \mathrm{~A}$
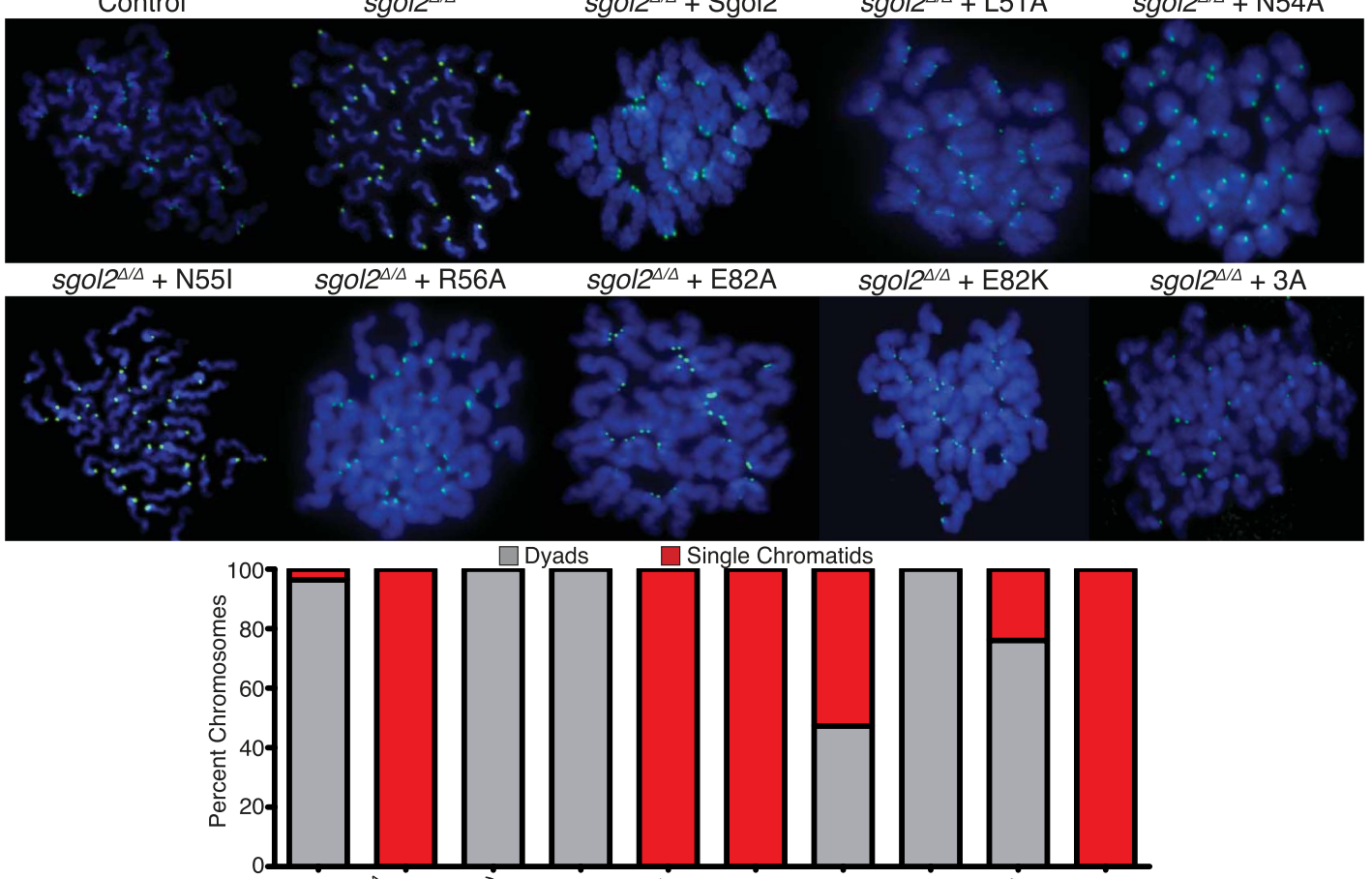

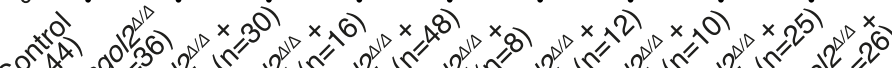

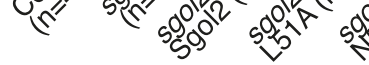

Figure 2. Sgol2-PP2A interaction is required to recruit PP2A to kinetochores and protect centromeric cohesion at the first meiotic division. (A) GV oocytes were harvested in the presence of IBMX. Microinjections were performed at GV stage in M2 medium supplemented with IBMX. Oocytes were then cultured in IBMX-free medium for about 6 hr, corresponding to metaphase I stage. Chromosome spreads were prepared and stained with DAPI Figure 2. Continued on next page 
Figure 2. Continued

(blue), Sgol2 (red), and PP2A-C (green). Box and whisker plot shows fluorescence intensity ratios of PP2A and Sgol2 at kinetochores. Upper and lower bars indicate 95th and 5th percentiles, respectively. Numbers of oocytes examined are indicated (n). (B) Oocytes harvested at GV stage were matured in M16 medium for up to $24 \mathrm{hr}$. Chromosome spreads prepared from oocytes that have extruded the first polar body were stained with DAPI (blue) to visualize DNA and CREST (green) to mark centromeres. Frequencies of dyads and single chromatids were quantified.

DOI: 10.7554/eLife.01133.004

that binding of PP2A to Sgol2's coiled coil is necessary for recruiting the phosphatase to kinetochores in vivo. Chromosome spreads revealed that the failure of $s g o / 2^{\Delta / \Delta}$ oocytes to maintain cohesion between sister centromeres after meiosis I was reversed by microinjection of wild type but not N54A, N55I, or 3A mRNAs (Figure 2B). L51A and E82A, which had little or no effect on PP2A's binding to Sgol2's coiled coil in vitro, also fully restored cohesion protection while R56A and E82K, which caused a modest reduction in the Sgol2cc-PP2A interaction vitro, only partially restored it, producing metaphase II oocytes containing individual chromatids as well as dyads (Figure 2B). Lastly, we noticed that injection of 3A Sgol 2 mRNAs also produced centromeric cohesion defects in wild type oocytes (Figure $3 A, B$ ). The correlation between the effects of mutations on binding PP2A in vitro and on protecting cohesion in vivo implies that the interaction of PP2A with Sgol2's coiled coil is essential for protecting cohesin from separase at the first meiotic division.

\section{Sgol2 silences the spindle assembly checkpoint by binding PP2A and Mad2}

DIC video microscopy revealed that sgol2 $2^{\Delta / \Delta}$ oocytes extruded polar bodies on average $2 \mathrm{hr}$ later than oocytes from wild type littermates. Moreover, only $61 \%$ of sgol2 $2^{\Delta / \Delta}$ oocytes extruded the first polar body compared with $88 \%$ in wild type controls (Figure 4A). To investigate this, wild type and sgol2 $2^{\Delta / \Delta}$ oocytes were microinjected with mRNA encoding histone H2B-mCherry to mark chromosomes and Securin-eGFP to measure APC/C ${ }^{\mathrm{Cdc} 20}$ activity (Figure $4 \mathrm{~B}$ ). $40 \%$ of mutant oocytes failed to activate APC/C $\mathrm{C}^{\mathrm{Cdc} 20}$ compared to $12 \%$ in wild type controls (Figure $4 \mathrm{C}$ ), and those that did so were delayed by about $2 \mathrm{hr}$ (Figure 4D, Figure 4-figure supplement 1). Surprisingly, microinjection of Sgol2 mRNA not only increased the fraction of oocytes that activated APC/C Cdc20 to wild type levels (89\%), but also advanced activation so that it took place about $2 \mathrm{hr}$ earlier than in wild type (Figure 4E-G, Figure 4-figure supplements 2 and 3). Because microinjection of mRNA encoding a version of Cdc20 (R132A) that cannot bind Mad2, but not wild type Cdc20, induced securin destruction in all sgol2 ${ }^{\Delta / \Delta}$ oocytes (Figure $4 C$ ), we conclude that Sgol2 facilitates APC/C ${ }^{C d c 20}$ activation by silencing the $\mathrm{SAC}$ and that this process is dose dependent.

The recent observation that shugoshins, like Sgol2 from humans and Sgo1 from Xenopus, bind Mad2 in a manner similar to Mad1 and Cdc20 through a conserved Mad2 Interaction Motif (MIM) (Orth et al., 2011) provided a clue as to how Sgol2 might regulates the SAC. This motif is conserved in mouse Sgol2 (Figure 1C) and far-western blots confirmed that interaction between $\mathrm{mSgol} 2$ and Mad2 is weakened by substituting the basic amino acid at its center with alanine (H150A) or glutamic acid (H150E) (Figure 1G). Strikingly, H150E and H150A Sgol2 mRNAs were less effective than wild type in increasing the fraction of sgol2 ${ }^{\Delta / \Delta}$ oocytes that activated APC/C ${ }^{C d c 20}$ (Figure 4E). They also failed to advance the timing of activation (Figure 4F, Figure 4-figure supplement $2 B, C$ ). Importantly, neither mutation affected Sgol2's association with kinetochores, its ability to recruit PP2A (Figure 3C) or MCAK (Figure 5A) to this location, or protection of centromeric cohesion (Figure 3D). Interestingly, N54A, which reduces PP2A binding, also abrogated Sgol2's ability to promote APC/C ${ }^{C d c 20}$ activation (Figure 4E,F, Figure 4-figure supplement 2A,D) without having any effect on MCAK recruitment (Figure 5A). We conclude that Sgol2 has an important role in silencing the SAC during meiosis I by binding directly both to Mad2 and PP2A.

\section{Sgol2's recruitment of MCAK regulates bivalent stretching}

We noticed that chiasmata appeared terminalized in a large fraction of bivalent chromosomes from sgol2 ${ }^{\Delta / \Delta}$ oocytes (Figure 5A). Because this effect was not accompanied by any reduction of cohesin along inter-chromatid axes (Figure 5B), it is unlikely to be caused by precocious Rec8 cleavage. Live cell imaging of oocytes microinjected at the GV stage with mRNAs encoding H2B-mCherry and GFP-MCAK showed that maternal and paternal kinetochores were pulled further apart in $5 g \circ / 2^{\Delta / \Delta}$ oocytes at metaphase I ( $t$ test, $p<0.0001)$. In other words, bivalents were stretched more and possibly the 


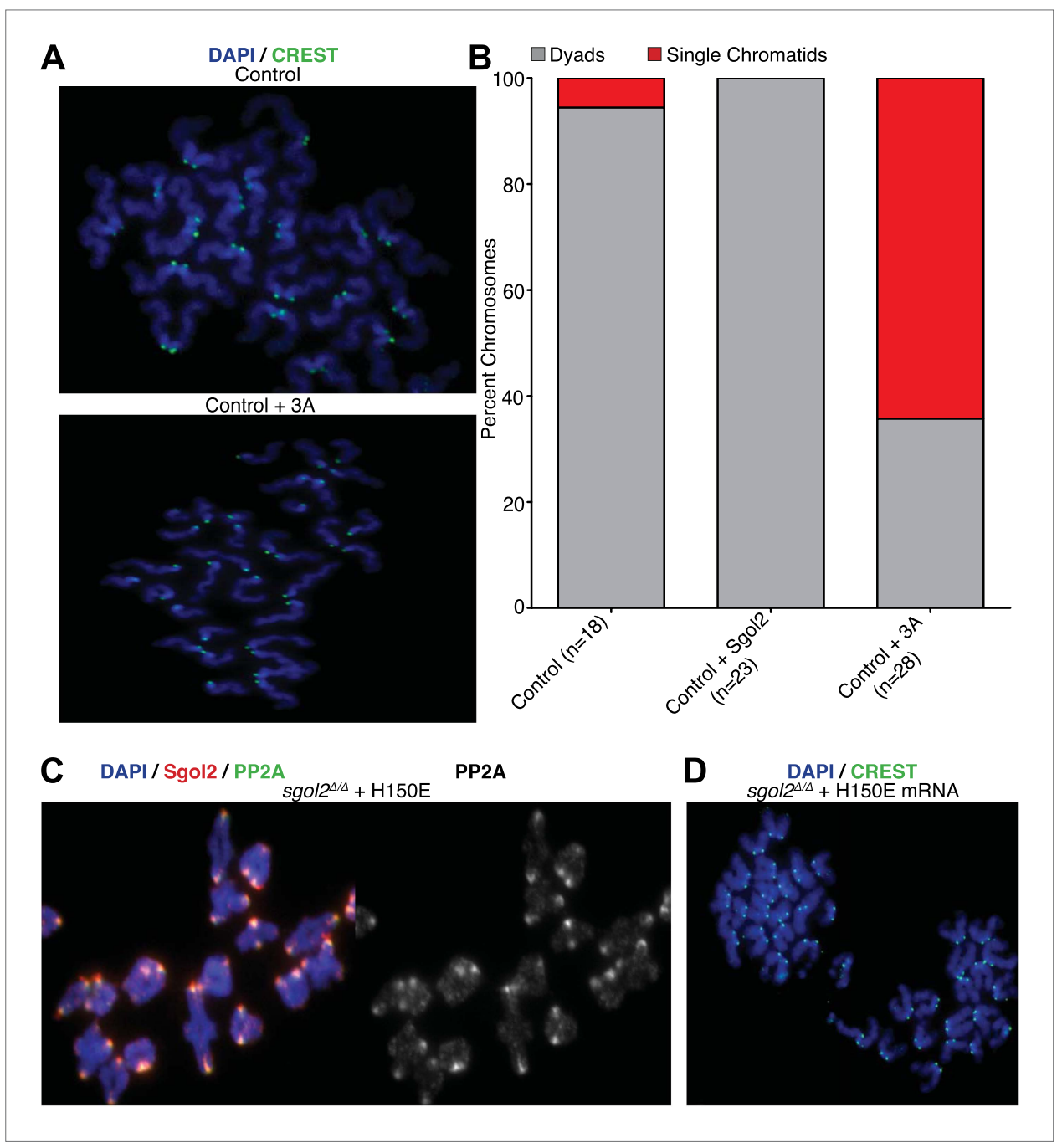

Figure 3. Sgol2-PP2A, but not Sgol2-Mad2, interaction is required to protect centromeric cohesion at the first meiotic division. (A) Oocytes harvested at GV stage from wild type (control) mice were microinjected with wild type or 3A mutant Sgol2 mRNA in M2 medium supplemented with IBMX. After releasing the oocytes in M16 medium without IBMX, chromosome spreads were prepared from metaphase II-arrested oocytes and stained with DAPI (blue) and CREST (green). (B) Frequencies of dyads and single chromatids observed on metaphase ll-stage chromosome spreads. Numbers of oocytes examined are indicated (n). (C) Metaphase I localization of Sgol2 (red) and PP2A-C (green) on chromosomes from sgol2 ${ }^{\Delta / \Delta}$ oocytes microinjected with H150E Sgol2 mRNA. (D) DAPI (blue) and CREST (green) stained chromosome spreads prepared from metaphase II-stage oocytes. DOI: 10.7554/eLife.01133.005

stretching of distal parts of bivalent chromosomes resulted in increased separation of homologous pairs on chromosome spreads. Importantly, increased kinetochore separation in sgo/2 $2^{\Delta / \Delta}$ oocytes was invariably observed on bivalents that had bi-oriented (Figures 5C and 6; see also Videos 1 and 2), but was not accompanied by any change in the overall length of metaphase I spindles (ANOVA, $p=0.97$; Figure 5D). Interestingly, microinjection of Sgol2 mRNA not only rescued chiasmata structure (Figure 5A), but also reduced inter-kinetochore distances to below that seen in wild type (Figures $5 \mathrm{C}$ and 6; Video 3, $t$ test $p<0.0001$ ), without altering spindle length (Figure 5D). Because a similar reduction in inter-kinetochore distances was observed with N54A or H150E mutations (Figures $5 \mathrm{C}$ and 6; Videos 4 and $5, t$ test $\mathrm{p}<0.0001$ ), regulation of bivalent stretching by Sgol2 does not depend on its ability to bind PP2A or Mad2. Thus, the effect cannot be due to changes either in arm cohesion protection or duration of metaphase I.

Inter-kinetochore distances correlated inversely with GFP-MCAK signals at kinetochores. Thus, MCAK is missing from kinetochores in sgol $2^{\Delta / \Delta}$ oocytes and more abundant (even than wild type) in mutant cells microinjected with Sgol2 mRNA (Figures 5A,C and 6). Because phosphorylation of human Sgol2 at 
A

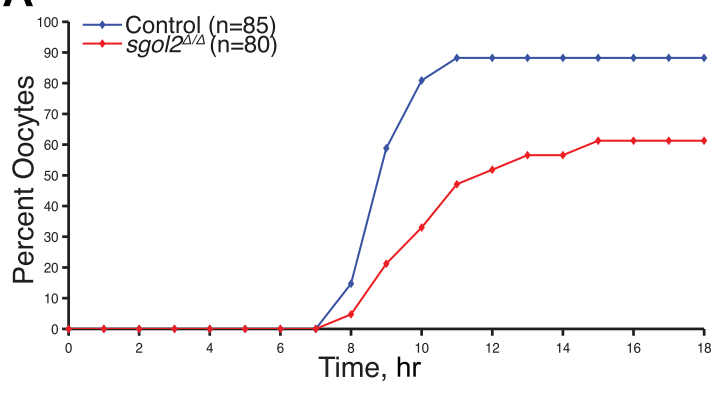

B

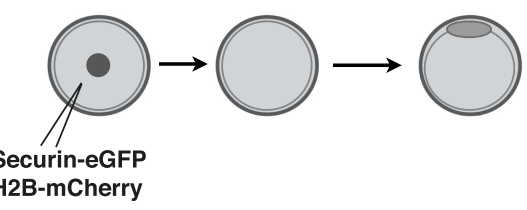

\section{C}

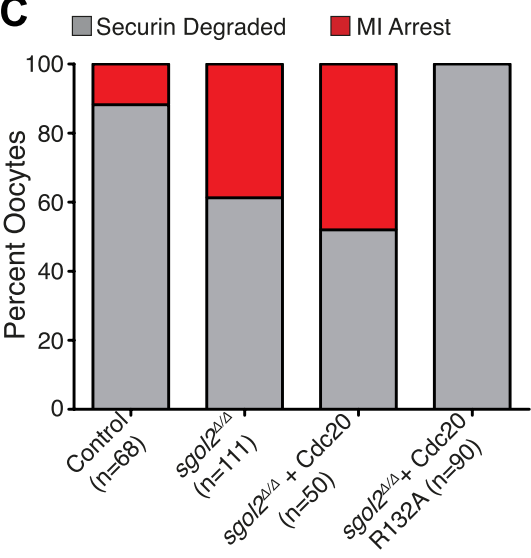

D

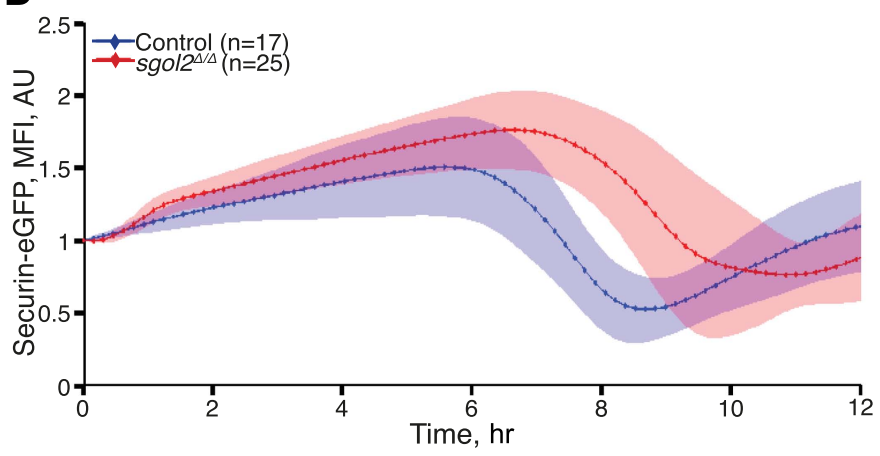

E $\square$ Securin Degraded

$\mathbf{F}$
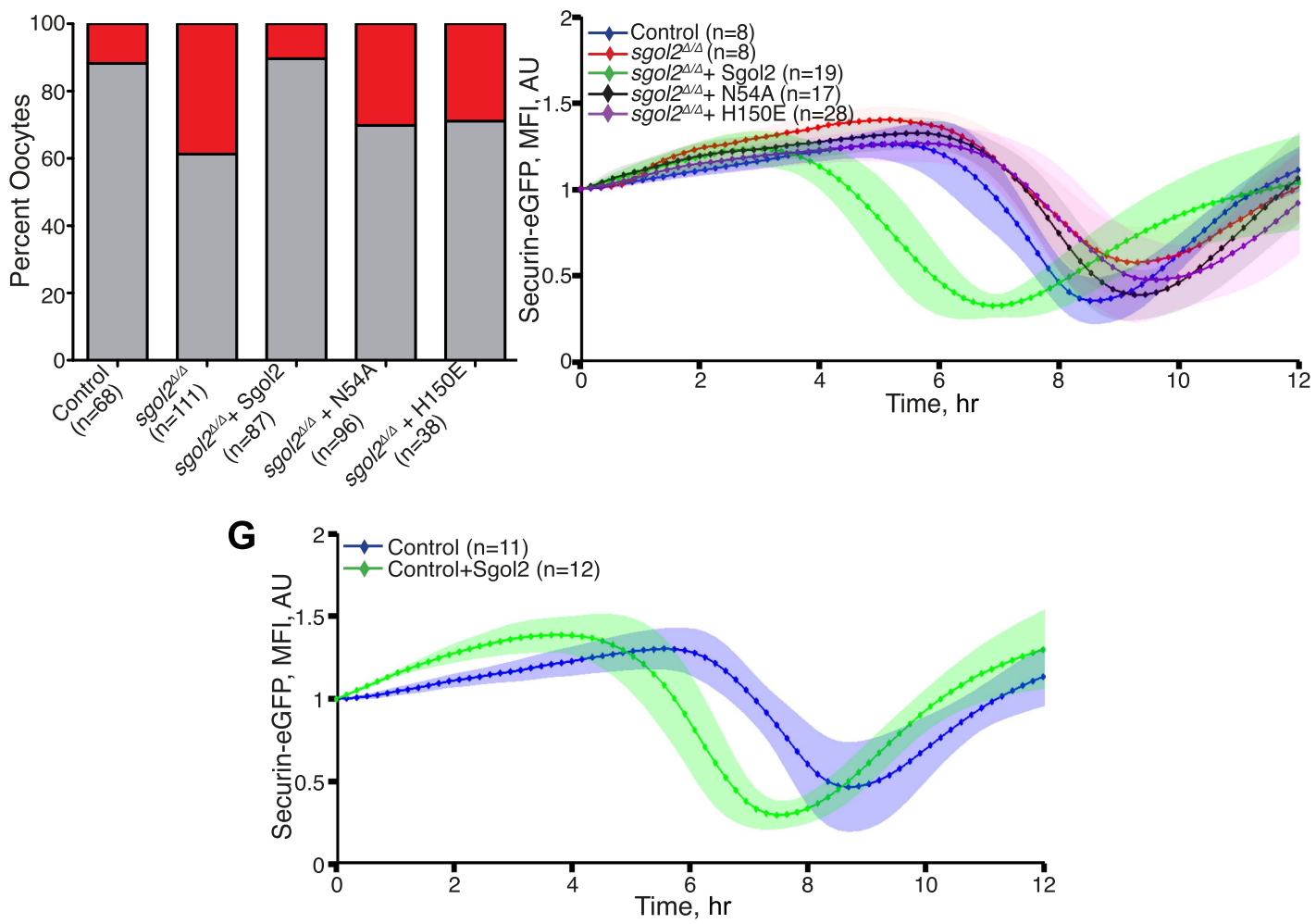

Figure 4. Sgol2 silences spindle assembly checkpoint through its interactions with PP2A and Mad2. (A) Kinetics of the first polar body extrusion (PBE) of oocytes cultured in vitro. Numbers of oocytes examined are indicated (n). (B) Oocytes were microinjected with indicated mRNAs at the GV stage. Chromosome movements and kinetics of Securin-eGFP were visualized by time-lapse confocal microscopy. (C) Frequencies of oocytes that destroyed Figure 4. Continued on next page 
Figure 4. Continued

Securin-eGFP, hence activated APC/C Cdc20, at the metaphase-anaphase transition are shown. SAC was inactivated by expressing a dominant-negative version of the APC/C activator Cdc20 (Cdc20-R132A) that cannot be bound by Mad2. (D) Data represent the mean and standard deviations of Securin-eGFP mean fluorescence intensity levels in sgol2 ${ }^{\Delta / \Delta}(\mathrm{red})$ and litter mate control (blue) oocytes at each time point. Values from individual oocytes were normalized relative to that at GVBD $(0 \mathrm{hr})$, and mean and standard deviations of the population are plotted in arbitrary units, (AU), against time. Securin-eGFP curves from individual oocytes are shown in Figure 4-figure supplement 1. (E) Securin-eGFP destruction was used as a marker for APC/C activation. Control and Sgol2 knockout are compared to sgol2 ${ }^{\Delta / \Delta}$ oocytes microinjected with indicated Sgol2 mRNA. (F) GV-normalized mean and standard deviations of Securin-eGFP levels are plotted against time for the indicated groups of oocytes. Securin-eGFP curves from individual oocytes are displayed in Figure 4-figure supplement 2. (G) Microinjection of Sgol2 mRNA accelerates APC/C Cdc20 activation in wild type oocytes. Mean and standard deviations of the time course measurements of Securin-eGFP mean fluorescence intensity are displayed. Securin-eGFP curves from individual oocytes are shown in Figure 4-figure supplement 3.

DOI: 10.7554/eLife.01133.006

The following figure supplements are available for figure 4:

Figure supplement 1. Time course measurements of Securin-eGFP mean fluorescence intensity in control and Sgol2 knockout oocytes. DOI: 10.7554/eLife.01133.007

Figure supplement 2. Comparison of Securin-eGFP mean fluorescence intensity curves in Sgol2 knockout oocytes and sgol2 ${ }^{\Delta / \Delta}$ oocytes expressing the indicated form of Sgol2 $2^{\Delta / \Delta} \mathrm{mRNA}$.

DOI: 10.7554/eLife.01133.008

Figure supplement 3. Microinjection of Sgol2 mRNA accelerates APC/C ${ }^{\text {Cdc20 }}$ activation in wild type oocytes.

DOI: 10.7554/eLife.01133.009

T537 and T620 (corresponding to T521 and T600 on M.m Sgol2) by Aurora B has been implicated in the association of MCAK with Sgol2 in mitotic cells (Tanno et al., 2010), we tested the effect of AZD1152, an Aurora B (and C) kinase inhibitor. This eliminated accumulation at kinetochores of both T521 phosphorylated Sgol2 (Figure 7B) and MCAK (Figure 5A, Figure 5-figure supplement 1). Crucially, injection of mRNA encoding T521A T600A Sgol2 into sgol2 ${ }^{\Delta / \Delta}$ oocytes failed to restore accumulation of MCAK at kinetochores (Figure 5A, Figure 5-figure supplement 1) and to reduce inter-kinetochore distances (Figures $5 \mathrm{C}$ and 6; Video 6, $t$ test $\mathrm{p}=0.027$ ). Interestingly, neither the Aurora B/C inhibitor nor T521A T600A prevented MCAK's recruitment to chromosome arms (Figure 5-figure supplement 1). The effect of T521A T600A on MCAK's kinetochore binding was highly specific as the mutant Sgol2 protein still accumulated at kinetochores (albeit to a lesser extent), recruited PP2A to this location, and protected centromeric cohesion from separase (Figure 5E,F).

These observations suggest that bivalent stretching might be inhibited by recruitment of MCAK to kinetochores by Sgol2. If so, increased inter-kinetochore distances in sgol $2^{\Delta / \Delta}$ oocytes should be reduced by targeting MCAK to centromeres by artificial means, namely by expression of an MCAK protein whose $\mathrm{N}$-terminus is fused to the C-terminus of CENPB-eGFP. Remarkably, CENPB-eGFPMCAK, but not CENPB-eGFP, reduced inter-kinetochore distances (Figure 5C, Figure 5-figure supplement 2; Video 7, $t$ test $p<0.0001$ ). We conclude that Sgol2 moderates the pulling force exerted on bivalents by recruiting MCAK to kinetochores, a process dependent on phosphorylation of Sgol2 by Aurora B/C kinases.

\section{Sgol2 promotes chromosome congression and bi-orientation}

The period between Germinal Vesicle Breakdown (GVBD) and anaphase can be divided according to the state of spindles and chromosomes into four distinct phases (Schuh and Ellenberg, 2007; Kitajima et al., 2011). During phase 1, multiple microtubule-organizing centers (MTOCs) form a ball of polymerizing microtubules on whose surface chromosomes are distributed individually. This is followed by a period (phase 2) in which chromosomes slide laterally over the ball shaped spindle apparatus, eventually congressing to form a 'prometaphase' belt around it. During phase 3, chromosomes invade the cluster of microtubules, which elongates along an axis perpendicular to the earlier chromosome belt, forming a barrel-shaped bipolar spindle. This highly dynamic phase culminates when chromosomes bi-orient on a metaphase plate within the bipolar spindle. During the fourth and final stage (phase 4), bi-oriented chromosomes oscillate around the metaphase plate with low amplitudes (Phase 4).

Live-cell confocal imaging of wild type mouse oocytes microinjected with H2B-mCherry and GFPMCAK showed that MCAK accumulates at MTOCs and on microtubules during phase 1 (Figure 6; Video 1) but does not appear at kinetochores until chromosomes congress on the surface of the ball at the start of phase 2. This event is accompanied by an increase in Sgol2's abundance at kinetochores 

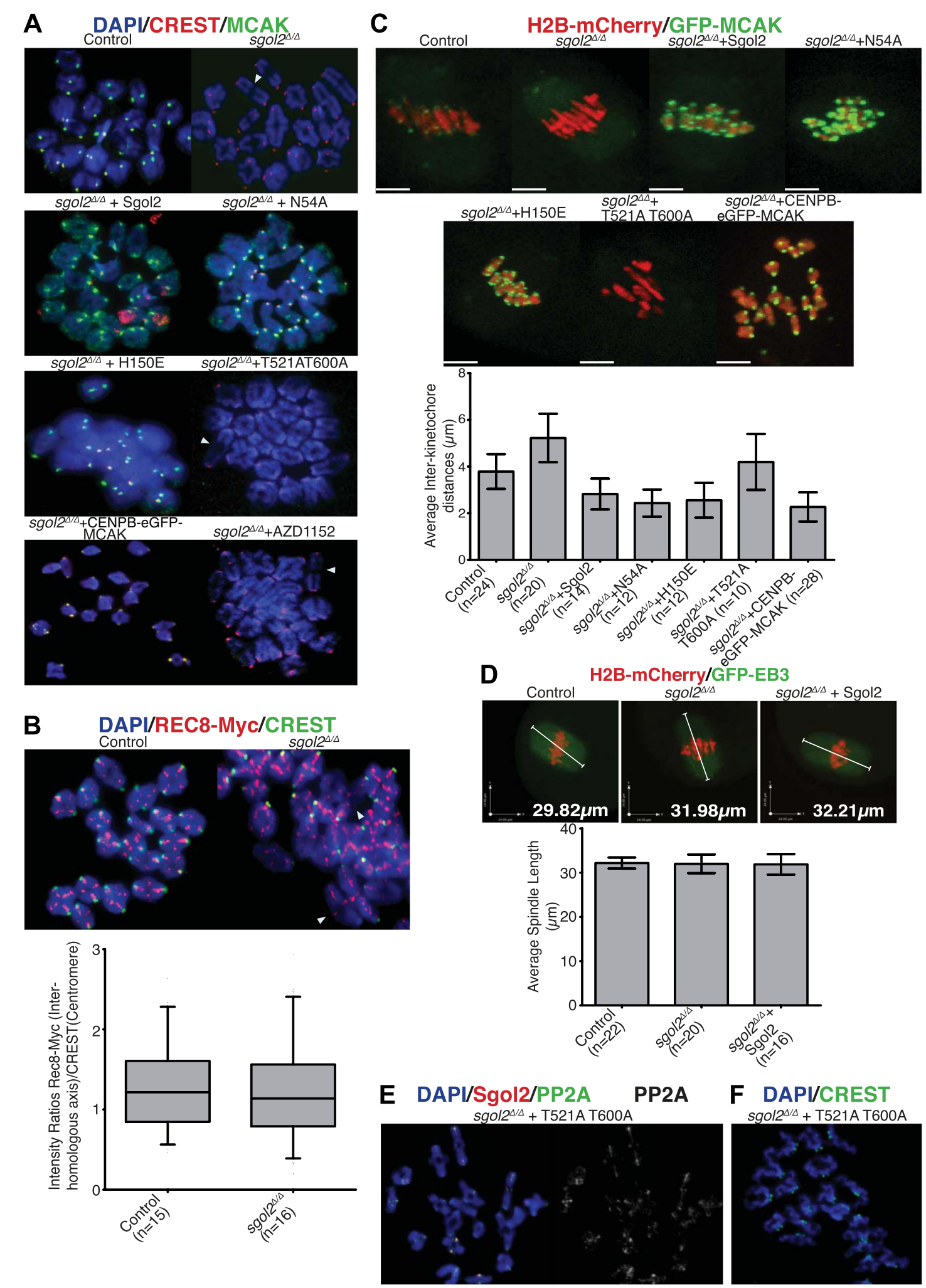

Figure 5. Sgol2, by recruiting MCAK to kinetochores, maintains inter-kinetochore distances between homologous chromosomes. (A) GV stage oocytes were harvested and microinjected in M2 medium supplemented with IBMX. Release from IBMX resulted in GVBD and at 6-7 hr post-GVBD chromosome spreads were prepared. Slides were stained with DAPI (blue), CREST (Red), and MCAK (green). Arrowheads indicate distally attached bivalent Figure 5. Continued on next page 
Figure 5. Continued

chromosomes. (B) Sgol2 targeted mice were crossed to (Tg)Rec8-Myc females to generate control and sgol2 ${ }^{\Delta / \Delta}$ expressing the Rec8-myc BAC transgene. Chromosome spreads were prepared at 6-7 hr post-GVBD and were stained with DAPI (blue), CREST (green), and c-Myc (Red). Intensity ratios of Rec8-Myc (on inter-kinetochore axes) and CREST (at centromeres) were calculated. (C) Representative Z-projected confocal microscopy images and average ( \pm SDs) inter-kinetochore distance measurements from metaphase I stage oocytes expressing H2B-mCherry (chromosomes, red) and GFP-MCAK (green). Scale bars represent $5 \mu \mathrm{m}$. (D) Z-projected (12 slices $1.5 \mu \mathrm{m}$ apart) live-cell confocal images and spindle length measurements $( \pm \mathrm{SDs})$ from metaphase I stage oocytes expressing EB3-GFP (microtubule plus ends, green) and H2B-mCherry (chromosomes, red). Scale bar is $14 \mu \mathrm{m}$. (E) Metaphase I localization of Sgol2 (red) and PP2A-C (green) on chromosomes from sgol2 ${ }^{\Delta / \Delta}$ oocytes microinjected with T521A T600A Sgol2 mRNA. (F) DAPI (blue) and CREST (green) stained chromosome spreads prepared at metaphase II-stage from sgol2 ${ }^{\Delta / \Delta}$ oocytes microinjected at GV stage with T521A T600A Sgol2 mRNA.

DOI: 10.7554/eLife.01133.010

The following figure supplements are available for figure 5 :

Figure supplement 1. GV oocytes were harvested in M2 medium supplemented with IBMX. DOI: 10.7554/eLife.01133.011

Figure supplement 2. Oocytes harvested at GV stage were microinjected with either CENPB-eGFP or CENPB-eGFP fused to MCAK mRNA. DOI: 10.7554/eLife.01133.012

and by phosphorylation of T521 (Figure 7A,B). MCAK's abundance at kinetochores gradually increases during phase 3 as chromosomes invade the spindle apparatus and peaks as chromosomes bi-orient on the metaphase plate (Figure 6, top panel; Video 1). This sequence of events is substantially altered in sgol2 ${ }^{\Delta / \Delta}$ oocytes. Though GFP-MCAK co-localizes with MTOCs and microtubules, it never subsequently accumulates at kinetochores (Figure 6). Chromosomes associate with the surface of the ball, albeit more loosely than in wild type, and undergo visible stretching instead of forming a prometaphase belt (Figures 6 and 8A, Figure 6-figure supplement 1; Video 2). This excessive stretching becomes even more pronounced upon formation of bipolar spindles. Thus, the clear demarcation of congression during phase 2 and bi-orientation during phase 3 was largely absent. Microinjection of Sgol2 mRNAs into sgol2 $2^{\Delta / \Delta}$ oocytes greatly accelerated both invasion of the ball by chromosomes and their bi-orientation, with the latter occurring even earlier than in wild type oocytes and before metamorphosis of the ball into a bipolar spindle (Figures 6 and 8A, Figure 6-figure supplement 1; Video 3). Thus, while loss of Sgol2 delays bi-orientation, the modest over-expression caused by mRNA microinjection accelerates this process.

Crucially, the precocious bi-orientation caused by Sgol 2 mRNA injection was unaffected by N54A or by H150E mutations but abrogated by T521A T600A (Figures 6 and 8A, Figure 6-figure supplement 1; Videos 4, 5, 6), which also abolished MCAK's accumulation at kinetochores and compromised chromosome alignment (Figures 5A and 6, Figure 6-figure supplement 1), possibly due to increased association of MCAK with the arms of bivalent chromosomes (Figure 5-figure supplement 1). Injection of Sgol2 T521A T600A still advanced polar body extrusion (Figure 6), demonstrating that SAC silencing by Sgol 2 does not depend on rapid or possibly even accurate chromosome bi-orientation and suggesting that these activities are mediated by separate domains within Sgol2. Surprisingly, injection of mRNAs encoding CENPB-eGFP-MCAK instead of Sgol2 did not accelerate chromosome bi-orientation (data not shown), raising the possibility that $\mathrm{Sgol} 2$ facilitates bi-orientation by a mechanism either independent of or in addition to MCAK's recruitment to kinetochores.

\section{Sgol2 regulates kinetochore-microtubule interactions}

Because, bi-orientation of bivalent chromosomes correlates with the appearance of kinetochoremicrotubule bundles (Kitajima et al., 2011), we visualized these in oocytes fixed in 1\% PFA following a brief treatment with a calcium-containing buffer, which destabilizes the more dynamic non-kinetochore-microtubules. This revealed three differences between wild type and sgol2 ${ }^{\Delta / \Delta}$ oocytes at $6 \mathrm{hr}$ post-GVBD, when most kinetochores in wild type have relinquished lateral and formed end on attachments and chromosomes have bi-oriented. Kinetochore associated microtubules bundles (K-fibers) were thinner and more sparse, lateral attachments were greatly increased in frequency, and kinetochores (as detected by CREST antibodies) were frequently highly stretched in mutant oocytes (Figure 8B). Microinjection of Sgol2 mRNA had the opposite effect, eliminating lateral attachments and kinetochore stretching while greatly increasing the abundance of end-on microtubules associated with each kinetochore, an effect abrogated by T521A T600A (Figure 8B). This suggests that the accumulation at kinetochores of Sgol2 phosphorylated by Aurora B/C kinase stimulates the conversion of lateral to end 


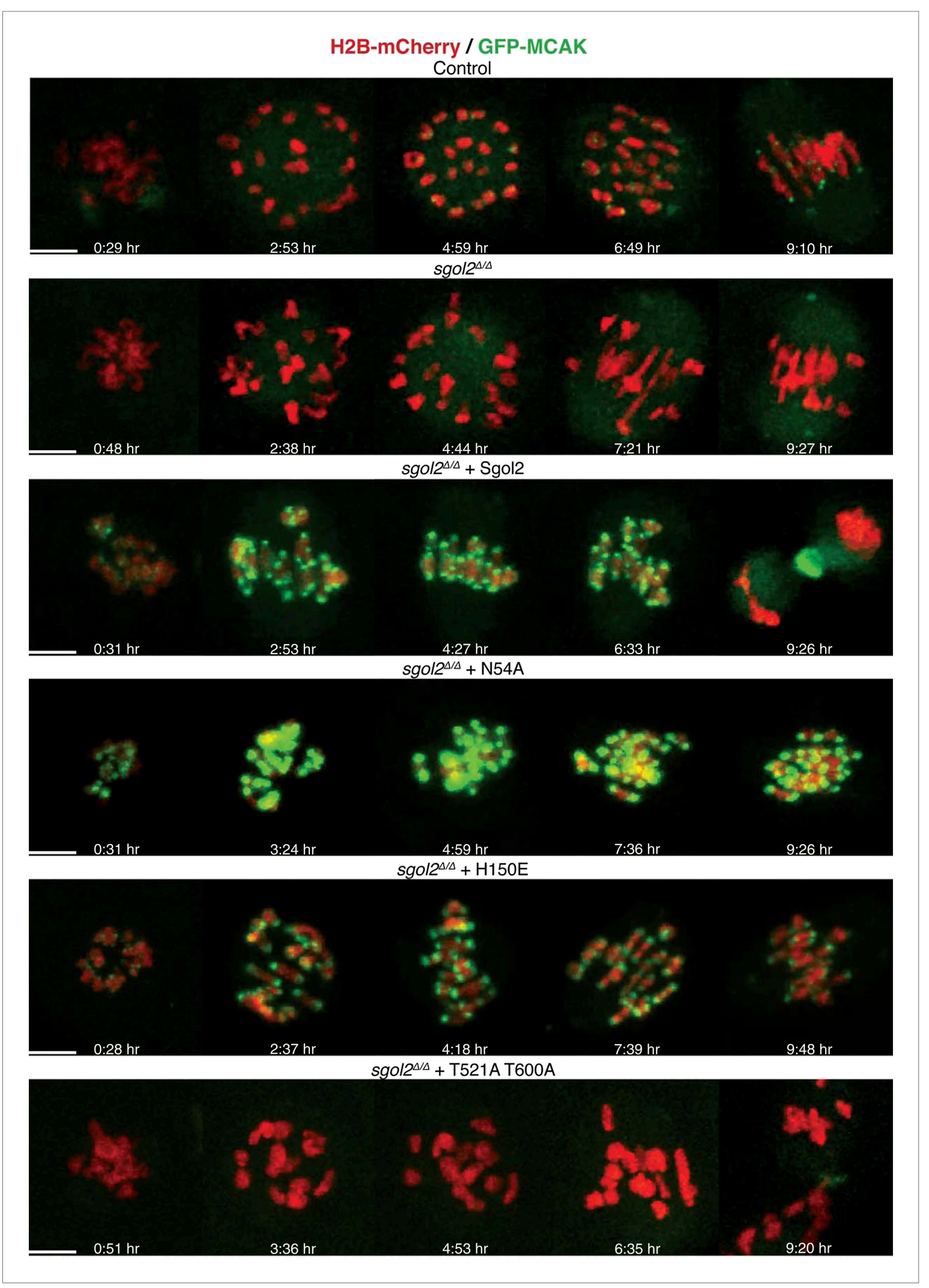

Figure 6. Sgol2 assists in bi-orientation of bivalent chromosomes on the metaphase plate. Time-lapse confocal microscopy images from oocytes expressing H2B-mCherry and GFP-MCAK. Representative images from litter-mate control, sgol2 ${ }^{\Delta / \Delta}$, sgol2 ${ }^{\Delta / \Delta}$ microinjected with wild type, N54A, H150E, Figure 6. Continued on next page 
Figure 6. Continued

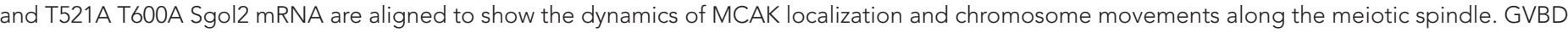
normalized time (in $\mathrm{hr}$ ) is indicated on each frame. Scale bars represent $5 \mu \mathrm{m}$.

DOI: 10.7554/eLife.01133.013

The following figure supplements are available for figure 6:

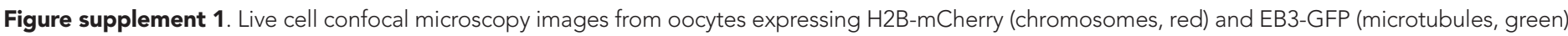
are shown

DOl: 10.7554/eLife.01133.014

on attachments and that this may be the mechanism by which Sgol2 accelerates chromosome biorientation. Because injection of CENPB-eGFP-MCAK mRNAs instead of Sgol2 largely failed to increase end-on attachments at the expense of lateral ones (Figure 8B), we suspect that Sgol2 phosphorylated by Aurora B/C has a function at kinetochores besides MCAK recruitment (see below).

\section{Sgol2 inhibits Aurora B/C kinase activity at kinetochores}

Because Aurora B kinase destabilizes kinetochore-microtubule attachments, at least partly by phosphorylating proteins within the KMN network (Welburn et al., 2010), we tested whether Sgol2 altered the phosphorylation of Ser24 on Knl1 (p-Knl1). Remarkably, the ratio of p-Knl1 to CREST signals in sgol2 ${ }^{\Delta / \Delta}$ oocytes at $6 \mathrm{hr}$ post-GVBD was more than double that of wild type controls (Figure 9A). Microinjection of Sgol2 mRNA reduced this to below wild type, an effect unaltered by N54A but largely abolished by T521A T600A (Figure 9A). Similar results were obtained using a phospho-specific antibody against Ser100 on Dsn1 (data not shown) and one that detects activating auto-phosphorylation of Aurora A/B/C at Thr288, Thr232, or Thr198 (p-Aurora), respectively (Figure 9B). Interestingly, these effects were specific to kinetochores as auto-phosphorylation of Aurora B/C on chromosome arms was unaltered in the mutant oocytes (Figure 9B). Importantly, Sgol2 did not alter the overall amount of Aurora $\mathrm{C}$ at kinetochores (Figure 10). These results imply that Sgol2 counters phosphorylation of KMN network proteins by a mechanism not involving its recruitment of PP2A. Strangely, this process seems to depend on Sgol2's prior phosphorylation by the very same kinases that it ultimately inhibits. In other words, Aurora B/C creates its own inhibitor at kinetochores, creating a negative feedback loop. Consistent with this notion, the gradual accumulation at kinetochores of Sgol2 phosphorylated at T521 following GVBD (Figure 7B) is accompanied by a corresponding reduction in Dsn1 Ser100 phosphorylation (Figure 9D). Critically, Sgol2's ability to inhibit Aurora B/C kinase activity at kinetochores does not depend on kinetochore-microtubule interactions, as Sgol2 reduced auto-phosphorylation of Aurora B/C even in the presence of micro-

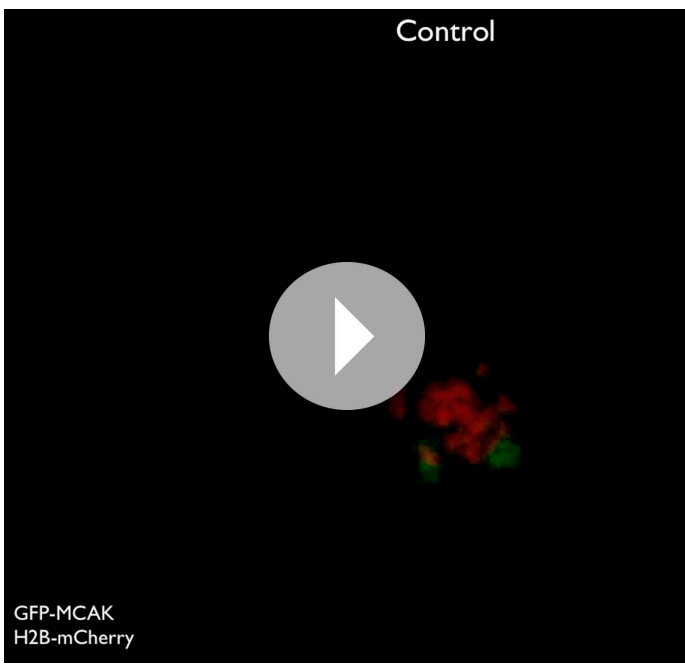

Video 1. Z-projected time-course confocal microscopy video of control oocyte microinjected at GV stage with GFP-MCAK and H2B-mCherry mRNA.

DOI: 10.7554/eLife.01133.015 tubule destabilizing drug (Figure 9C). To address whether hyper-activity of Aurora B/C at kinetochores could be responsible for the paucity of $\mathrm{K}$-fibers in sgol2 $2^{\Delta / \Delta}$ oocytes, we tested the effect of the Aurora kinase inhibitor AZD1152. This both increased the density of K-fibers and reduced kinetochore stretching but did not eliminate lateral attachments (Figure $8 \mathrm{~B}$ ), which may require direct involvement of MCAK. We suggest that Sgol2 promotes chromosome bi-orientation by inhibiting Aurora B/C kinase as well as by recruiting MCAK.

\section{Discussion}

Previous work has shown that Sgol2 is essential for protection of centromeric cohesin from separase at the first meiotic division (Lee et al., 2008; Llano et al., 2008). Our creation and analysis of mutations within Sgol2's highly conserved coiled coil reveals the likely mechanism, namely recruitment of PP2A to centromeres. PP2A might 


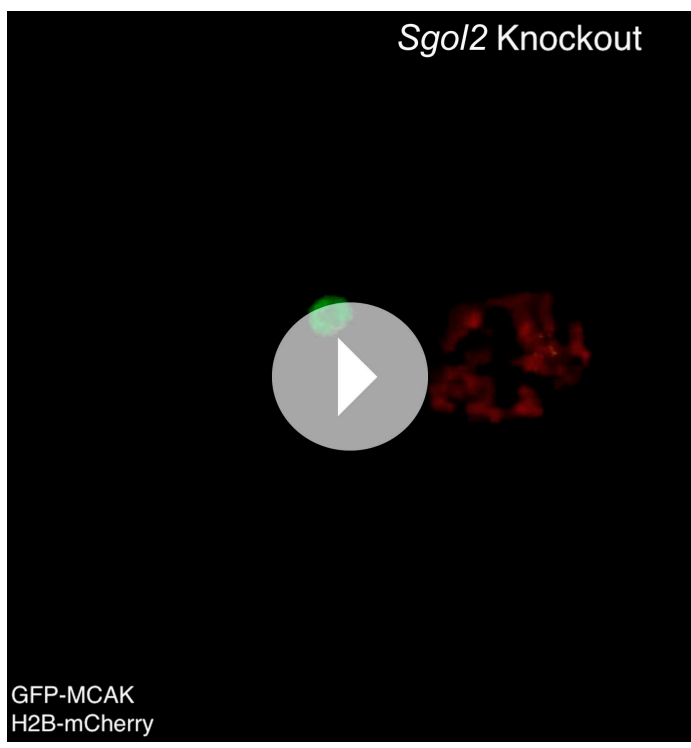

Video 2. Z-projected time-course confocal microscopy video of sgol2 $\Delta / \Delta$ oocyte microinjected at GV stage with GFP-MCAK and H2B-mCherry mRNA.

DOI: 10.7554/eLife.01133.016

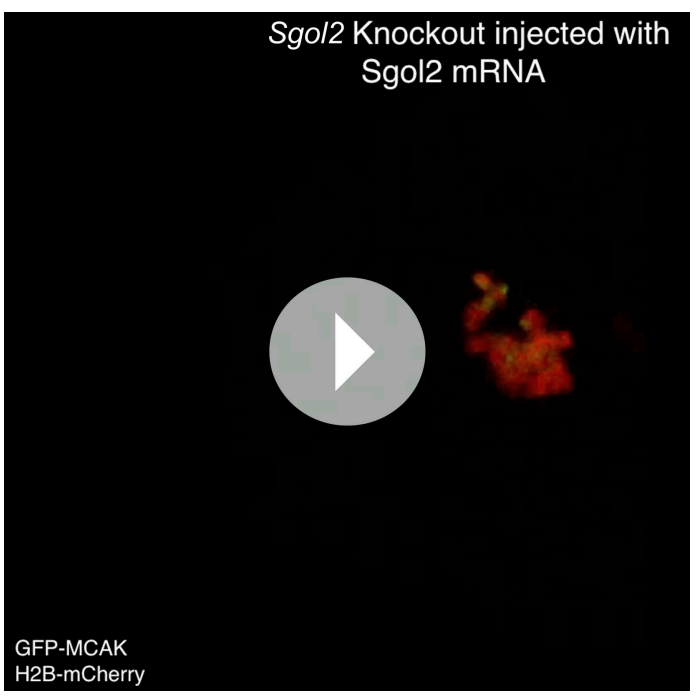

Video 3. Z-projected time-course confocal microscopy video of sgol2 ${ }^{\Delta / \Delta}$ oocyte microinjected at GV stage with GFP-MCAK, H2B-mCherry and Sgol2 mRNA.

DOI: 10.7554/eLife.01133.017

recruitment of MCAK to kinetochores by fusion to CENP-B substitutes for Sgol2 with regard to bivalent stretching, we suggest that it is the population of MCAK recruited to kinetochores by Sgol2 that moderates the pulling forces exerted on maternal and paternal kinetochores, possibly by reducing the incidence of lateral attachments.

Artificial MCAK recruitment did not however restore normal looking end on attachments or greatly facilitate bi-orientation in Sgol2 deficient oocytes, implying that Sgol2 has yet another function at kinetochores besides recruiting PP2A and MCAK. Our observation that auto-phosphorylation of Aurora B/C kinases as well as phosphorylation of KMN proteins are elevated in Sgol2's absence but depressed upon microinjection of Sgol 2 mRNA suggests that this other function of Sgol2 is to act 


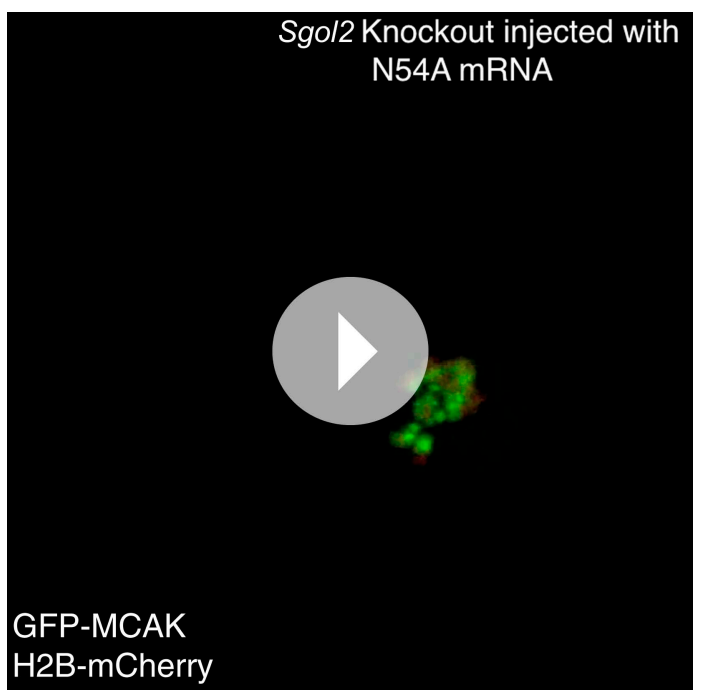

Video 4. Z-projected time-course confocal microscopy video of sgol2 ${ }^{\Delta / \Delta}$ oocyte microinjected at GV stage with GFP-MCAK, H2B-mCherry and N54A Sgol2 mRNA. DOI: 10.7554/eLife.01133.018

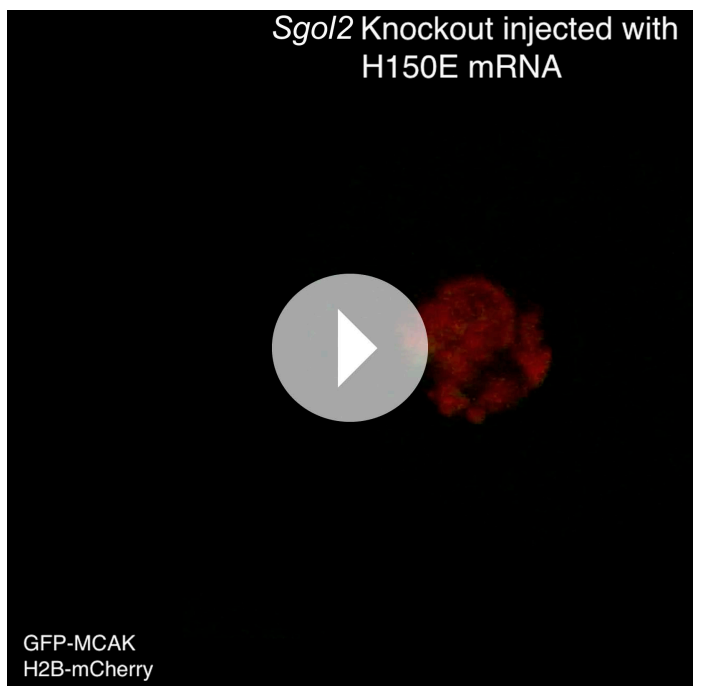

Video 5. Z-projected time-course confocal microscopy video of sgol2 ${ }^{\Delta / \Delta}$ oocyte microinjected at GV stage with GFP-MCAK, H2B-mCherry and H150E Sgol2 mRNA. DOI: 10.7554/eLife.01133.019

which Aurora B/C activates its own inhibitor. The regulatory network revealed by our work ensures that Aurora B/C kinases will be active early during the bi-orientation, when incorrect attachments may be more frequent, and less active later, when they have been largely eliminated. It is possible that during the period when Aurora B/C kinases are active, the selective stabilization of correct attachments is mediated by mechanical changes within kinetochores induced by tension, as recently observed in purified yeast kinetochores (Akiyoshi et al., 2010), not as originally thought by tension dependent changes in Aurora kinase activity, for which there has never been much direct evidence. We suggest that an important feature of meiosis I in oocytes is an early phase of high Aurora B/C kinase activity at kinetochores followed by a later phase of lowered activity made possible by the Aurora B/C-Sgol2 negative feedback loop. The lowered activity of Aurora B/C might increase the MT-depolymerizing activity of MCAK at kinetochores (Andrews et al., 2004). This is supported by the fact that the kinetochore pool of MCAK, recruited by Sgol2, is indeed not phosphorylated at serine 92 in mouse oocytes 


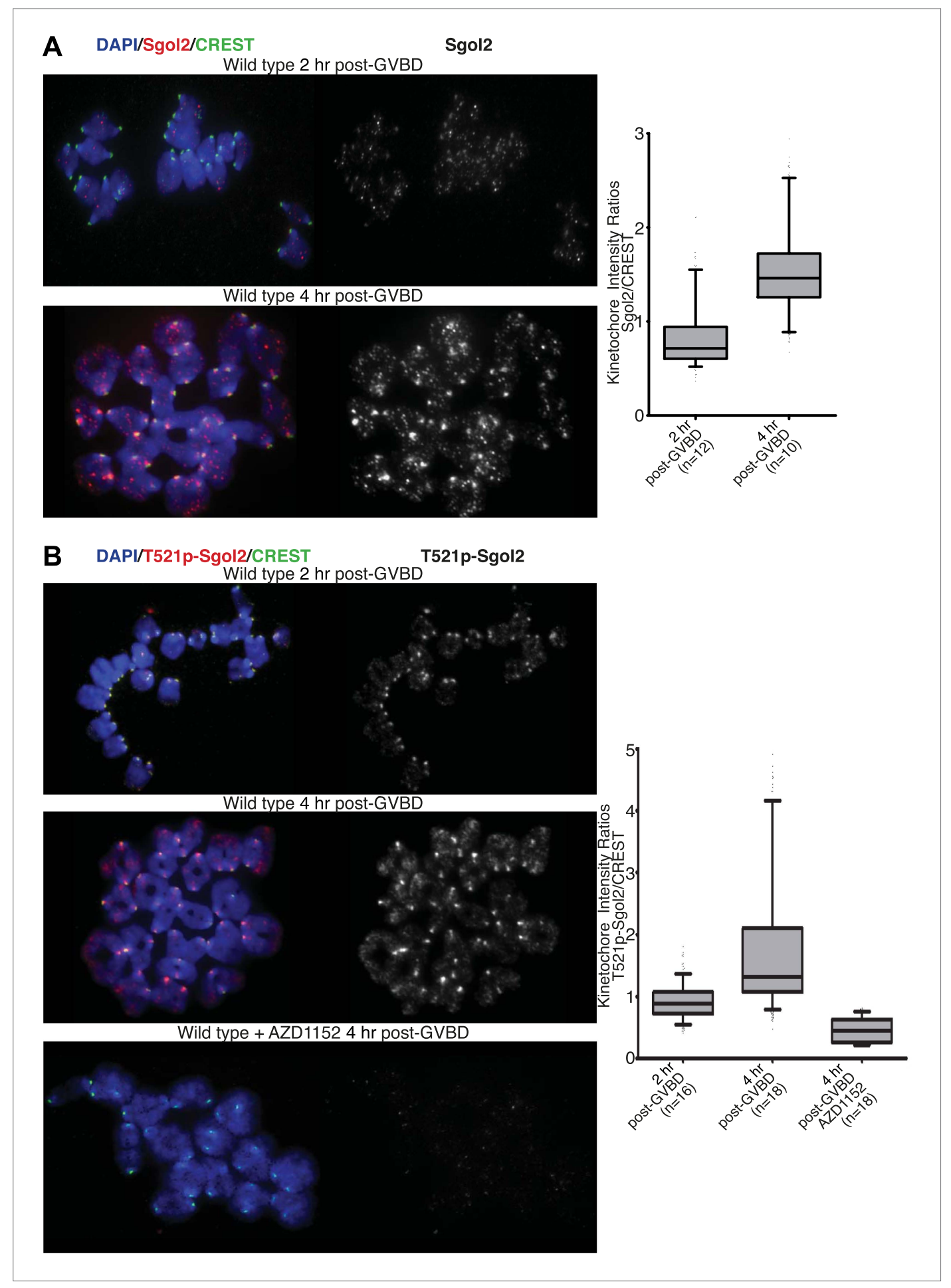

Figure 7. Aurora B/C Kinase phosphorylates Sgol2 on T521. (A) Oocytes harvested from wild type control females at GV stage were matured in vitro and chromosome spreads were prepared at 2 and $4 \mathrm{hr}$ post-GVBD. Slides were stained for DNA (blue), Sgol2 (red), and CREST (green). Fluorescence intensity ratios of Sgol2 and CREST at kinetochores were quantified. Numbers of oocytes examined are indicated (n). (B) Chromosome spreads were prepared at 2 and $4 \mathrm{hr}$ post-GVBD from wild type control oocytes. Slides were stained for DNA (blue), anti-T521p-Sgol2 (red), and CREST (green). Aurora B/C kinase activity was inhibited by culturing oocytes in M16 medium supplemented with the AZD1152 (100 nM) inhibitor. Fluorescence intensity ratios of T521p-Sgol2 and CREST at kinetochores were quantified.

DOI: 10.7554/eLife.01133.020 
(data not shown). If correct, it will be important to understand the dynamic properties of the feedback loop that ensures that Aurora B/C kinases do not turn themselves off precociously. It is currently unclear whether the Aurora B/C-shugoshin feedback loop is a general feature of meiotic cells or whether it is also a feature of mitotic cells. How Sgol2 inhibits Aurora B/C kinase activity at kinetochores is also unclear, in particular whether Sgol2 does this alone or only in conjunction with the recruitment of MCAK.

Given the number of fundamental processes regulated by Sgol2, it is surprising that $s g o / 2^{\Delta / \Delta}$ oocytes often manage to undergo the first meiotic division without generating massive aneuploidy. Their second meiotic division is of course completely defective due to the total absence of sister chromatid cohesion. We suggest that all of the processes regulated by Sgol 2 are fundamentally essential but that they are also regulated by Sgol2-independent pathways. In other words, the processes regulated by Sgol2 may be so fundamental that there are multiple path-

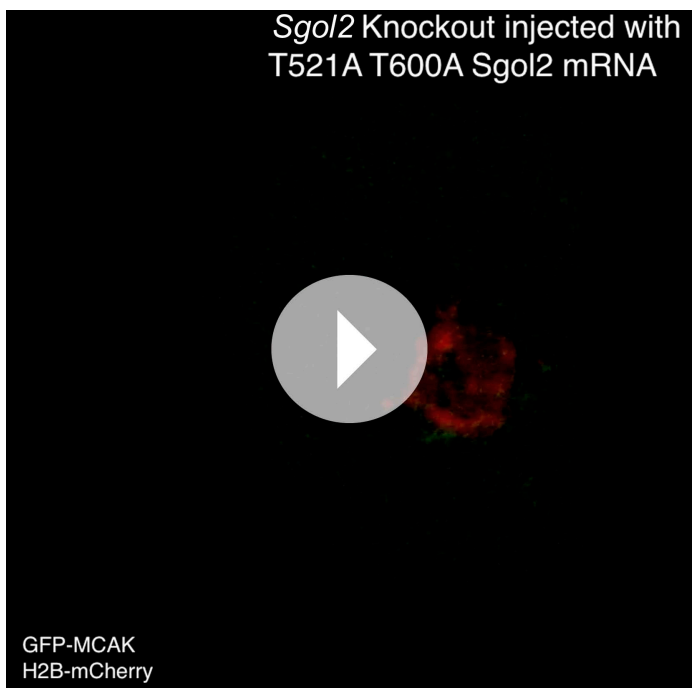

Video 6. Z-projected time-course confocal microscopy video of sgol $2^{\Delta / \Delta}$ oocyte microinjected at GV stage with GFP-MCAK, H2B-mCherry and T521A T600A Sgol2 mRNA.

DOI: 10.7554/eLife.01133.021

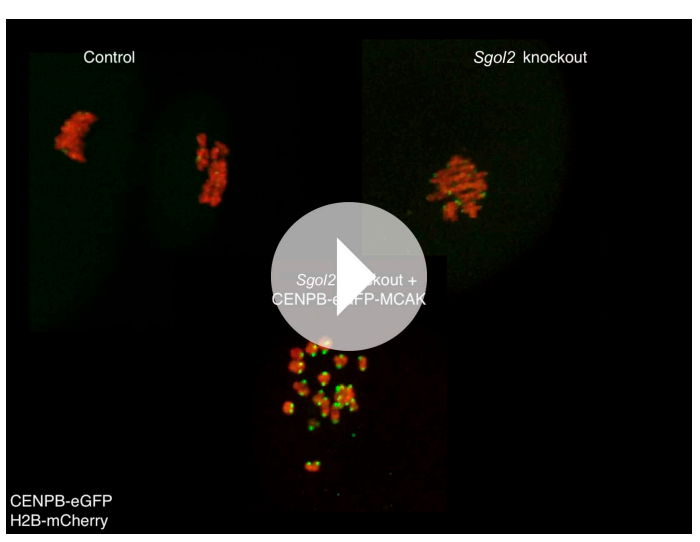

Video 7. Upper panel displays Z-projected timecourse confocal microscopy video of control and sgol2 ${ }^{\Delta / \Delta}$ oocytes microinjected at GV stage with CENPB-eGFP and H2B-mCherry mRNA. Lower panel displays representative confocal video of $\mathrm{sgol}^{\Delta / \Delta}$ oocyte microinjected with CENPB-eGFP-MCAK and H2B-mCherry mRNA

DOI: 10.7554/eLife.01133.022 ways regulating them. It is not inconceivable that Sgol1, which is also expressed during meiosis, shares some functions with Sgol2 and it will therefore be interesting to analyze the phenotype of double mutant oocytes.

What is possibly most remarkable is that a single protein regulates sister chromatid cohesion, chromosome traction, the spindle assembly checkpoint, and lastly Aurora B/C kinases. Crucially, by demonstrating that different point mutations alter the regulation of some but not other processes, we have proven that the pleiotropy observed in sgol $2^{\Delta / \Delta}$ oocytes is not due to knock on effects (secondary or even tertiary pathology) of eliminating a single function. Sgol2 possesses several different domains that regulate fundamentally different processes (Figure 11). Why so much regulation is embedded into a single protein is unclear, but we speculate that this helps the coordination of multiple processes necessary for high fidelity chromosome segregation.

\section{Materials and methods}

\section{Generation of mouse strains}

Generation of the Sgol2 knockout mice has been described (Llano et al., 2008). To detect Rec8, females from a transgenic line expressing Rec8 from a bacterial artificial chromosome (BAC), with nine tandem copies of the human c-myc epitope at its $C$ terminus, were crossed to $\mathrm{sgol}^{\mathrm{L} /+}$ males. Litters with genotypes sgol2 ${ }^{\Delta /+}(\mathrm{Tg}) \operatorname{Rec} 8-\mathrm{Myc}+$ were crossed back to $\mathrm{sgol} / 2^{\Delta /+}$ mice to generate sgol2 $2^{\Delta /+}(\mathrm{Tg}) \operatorname{Rec} 8-\mathrm{Myc}+$ (controls) and $\mathrm{sgol} 2^{\Delta / \Delta}$ (Tg)Rec8-Myc+ female.

\section{Isolation and culture of oocytes}

Prophase-arrested oocytes were harvested from ovaries excised from 8-12 week old female mice. Antral follicles were isolated using sterile insulin needles in M2 medium (Sigma-Aldrich, UK) supplemented with $200 \mu \mathrm{M}$ IBMX (Sigma-Aldrich). For intracellular microinjections, oocytes were placed in droplets of M2 medium with IBMX. For timelapse confocal imaging and in vitro culture prior 


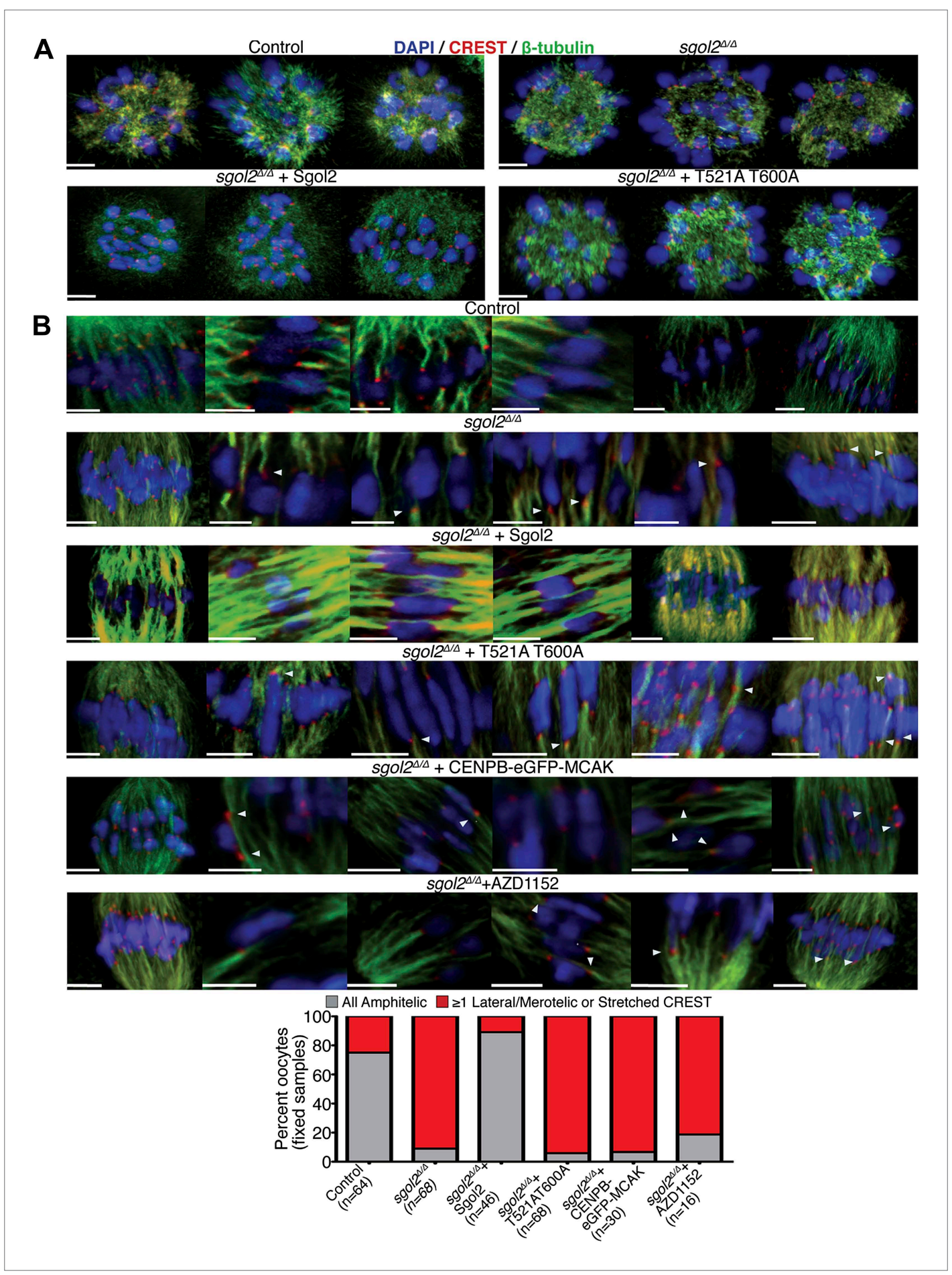

Figure 8. Sgol2 promotes bi-orientation of homologous chromosomes by increasing kinetochore-microtubule interactions. (A) To study the relative distributions of chromosomes on the ball shaped spindle, at 2-3 hr post-GVBD, oocytes were treated with $\mathrm{Ca}^{2+}$ buffer for $90 \mathrm{~s}$ and then fixed in $1 \%$ PFA. Cells were stained with anti- $\beta$-tubulin (microtubules, green), CREST (kinetochores, red), and DAPI (chromosomes, blue). Scale bars represent $5 \mu \mathrm{m}$. (B) Oocytes harvested at GV stage from wild type, sgol2 $2^{\Delta / \Delta}$ and sgol2 $2^{\Delta / \Delta}$ microinjected with wild type, T521A T600A Sgol2 mRNA and CENPB-eGFP-MCAK mRNA were cultured in M16 medium for $6 \mathrm{hr}$. At $6 \mathrm{hr}$ post-GVBD, oocytes were fixed in 1\% PFA were stained with anti- $\beta$-tubulin (microtubules, green), CREST (kinetochores, red), and DAPI (chromosomes, blue). For Aurora B/C inhibition, oocytes harvested from Sgol2 knockout females were cultured in M16 medium for 4 hr. At $4 \mathrm{hr}$ post-GVBD, oocytes were transferred to M16 medium supplemented with low concentration (20 nM) of AZD1152 inhibitor. Oocytes were cultured until $6 \mathrm{hr}$ post-GVBD and were then fixed in 1\% PFA. Scale bars represent $5 \mu \mathrm{m}$. DOI: 10.7554/eLife.01133.023 
A
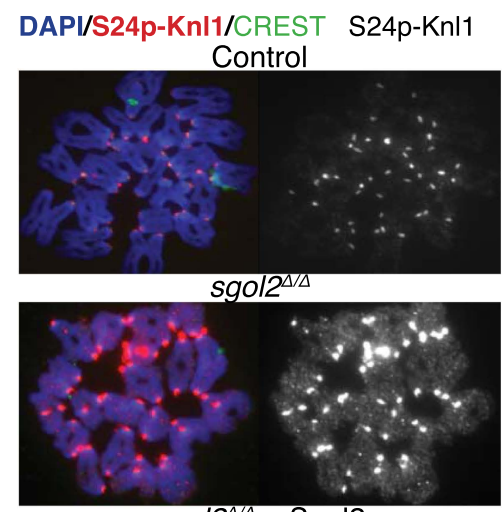

$\mathrm{sgol}^{\Delta / \Delta}+\mathrm{Sgol} 2$
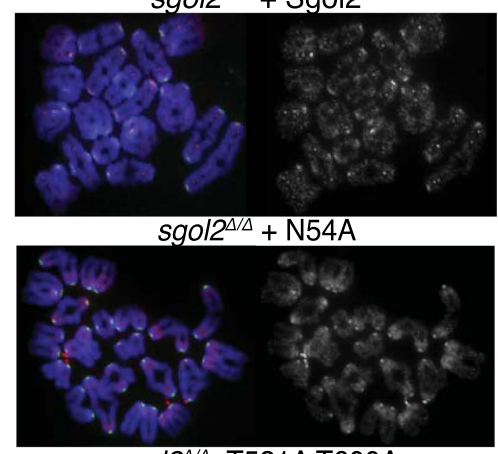

sgol2 $2^{\Delta / \Delta}+\mathrm{T} 521 \mathrm{~A} T 600 \mathrm{~A}$

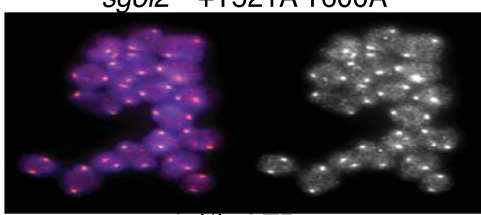

sgol2 $2^{\Delta / \Delta}+$ AZD1152
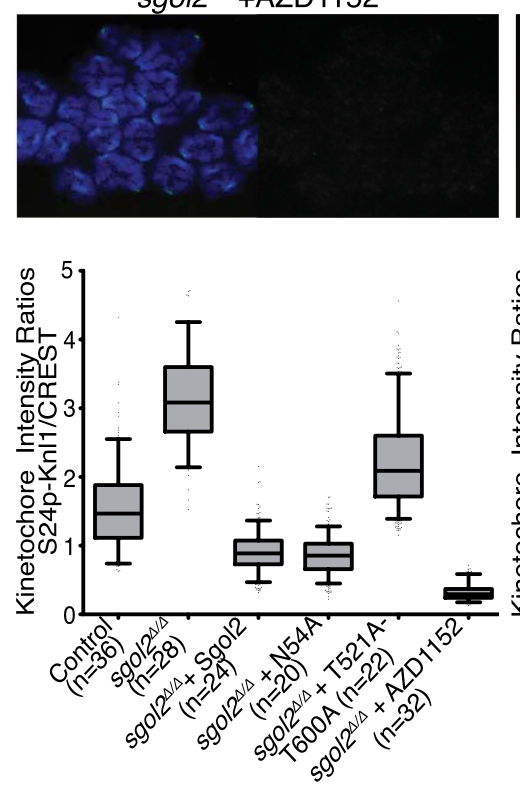

B
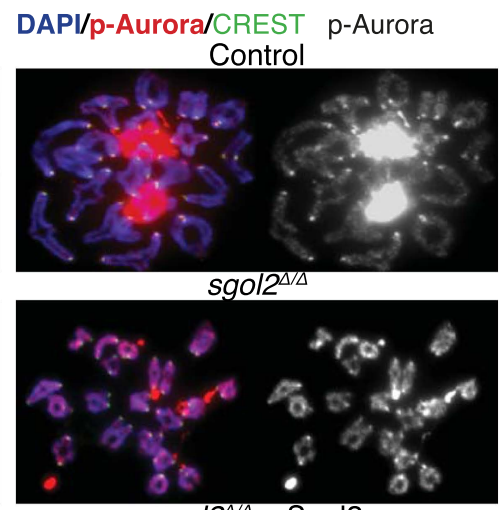

$\mathrm{sgol}^{\mathrm{\Delta} \Delta}+\mathrm{Sgol} 2$
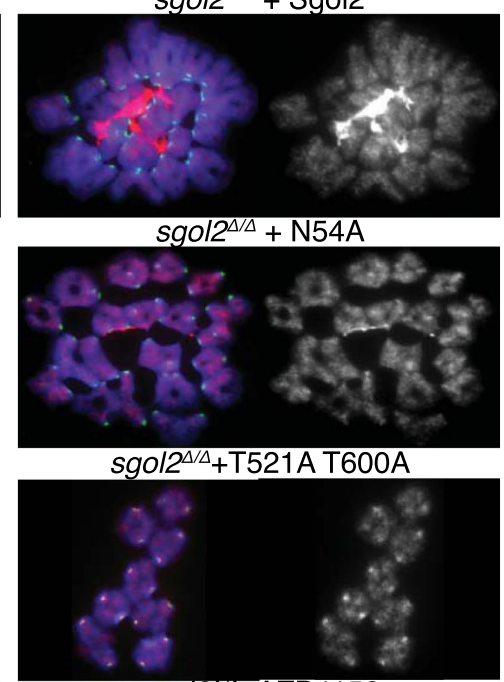

$s g o l 2^{\Delta / \Delta}+$ AZD 1152
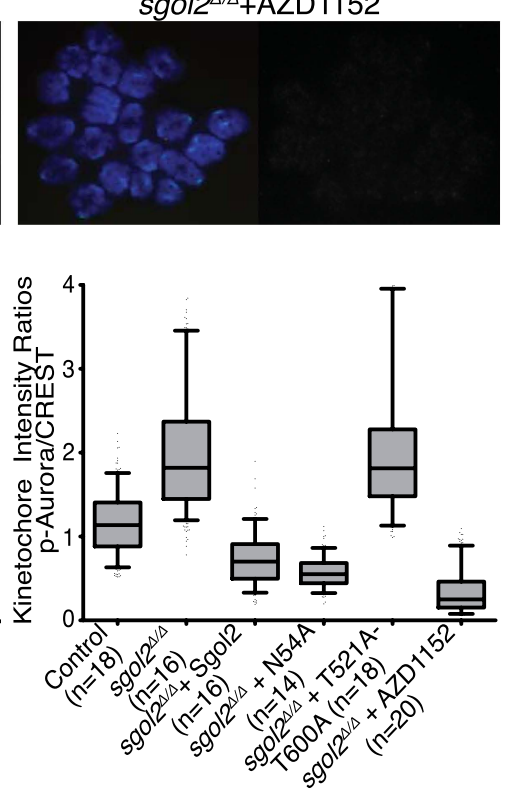

C

DAPI/p-Aurora/CREST p-Aurora

sgol2 $2^{\Delta / \Delta}$

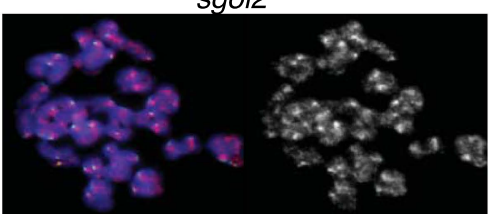

$\mathrm{sgol}^{\Delta / \Delta}+\mathrm{Sgol} 2$
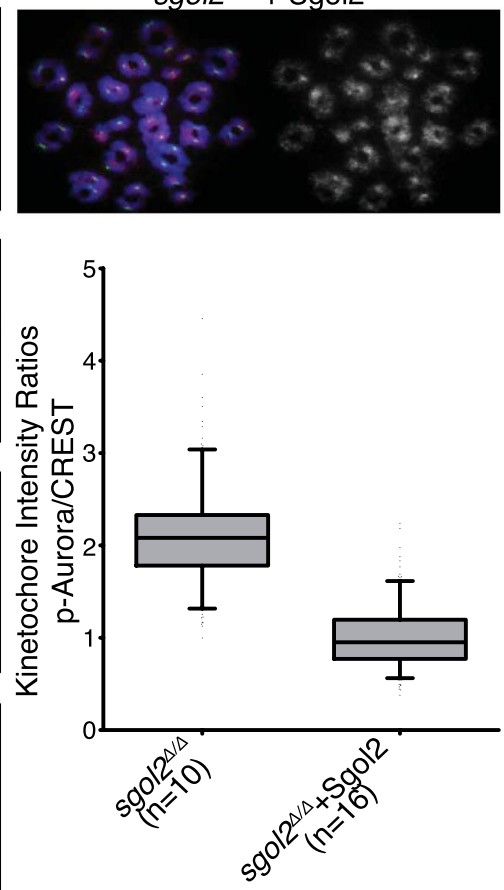

\section{D}

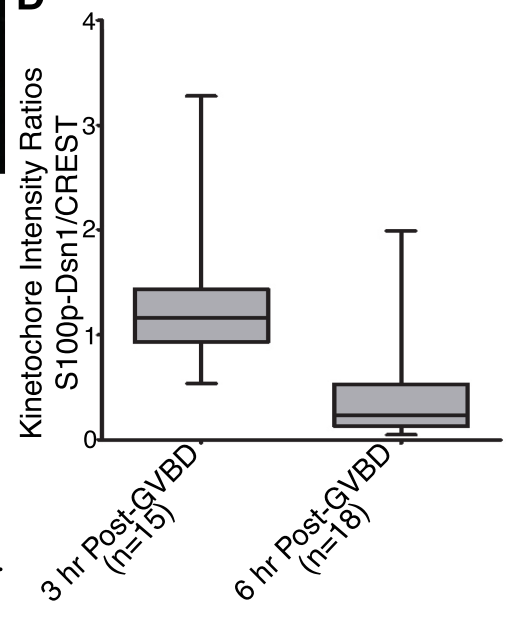

Figure 9. Sgol2 inhibits Aurora B/C Kinase activity at kinetochores. (A) Chromosome spreads were prepared from indicated groups at 4 hr post-GVBD. Slides were stained with DAPI (blue), anti-S24p-Knl1 (red) and CREST (green). Kinetochore intensities of anti-S24p-Knl1 and CREST signals were quantified and the intensity ratios of anti-S24p-Knl1 and CREST signals are shown. Upper and lower bars indicate 95th and 5th percentiles, respectively. (B) Chromosome spreads Figure 9. Continued on next page 
Figure 9. Continued

prepared at $4 \mathrm{hr}$ post-GVBD were stained with DAPI (blue), anti-p-Aurora A/B/C (red) and CREST (green). Kinetochore fluorescence intensity ratios of p-Aurora $\mathrm{A} / \mathrm{B} / \mathrm{C}$ and CREST are displayed. (C) Sgol2 knockout and Sgol2 knockout oocytes injected with wild type Sgol2 mRNA were cultured for 6 hr from GVBD in M16 medium supplemented with 400 nM Nocodazole. Chromosome spreads were stained for DNA (DAPI, blue), p-Aurora A/B/C (red), and CREST (green). (D) anti-S100p-Dsn1 and CREST kinetochore intensity ratios, quantified from chromosome spreads prepared at 3 and 6 hr post-GVBD from wild type control oocytes. DOI: 10.7554/eLife.01133.024

to preparation of chromosome spreads, oocytes were transferred into IBMX-free M16 medium and cultured at $37^{\circ} \mathrm{C}$ and $5 \% \mathrm{CO}_{2}$.

\section{Preparation of mRNAs}

Capped mRNA constructs with a poly-A tail were transcribed using T3 or T7 Ultra mMESSAGE kit (Ambion, Austin, TX, USA) from plasmid cDNA encoding H2B-mCherry, wild type Sgol2 (Origene, Rockville, MD, USA), and various mutants of Sgol2, GFP-Sgol2, Securin-eGFP, GFP-MCAK, CENPBeGFP, CENPB-eGFP-MCAK, and GFP-EB3.

\section{Microinjection of mRNA}

Fully-grown oocytes were injected with 5-10 pl mRNA at a final concentration of $0.1 \mathrm{mg} / \mathrm{ml}$ in RNase-free water (Ambion) using a Pneumatic PicoPump (World Precision Instruments). To allow for protein expression, following microinjection of mRNA, GV oocytes were cultured for $12 \mathrm{hr}$ in M16 supplemented with IBMX. Oocytes were then washed in inhibitor-free M16 and thereafter cultured at $37^{\circ} \mathrm{C}$ and $5 \% \mathrm{CO}_{2}$.

\section{Live cell confocal microscopy}

During time-lapse confocal microscopy experiments, oocytes were cultured in a PeCon environmental microscope incubator at $37^{\circ} \mathrm{C}$ and $5 \%$ CO2. A Zeiss LSM510 META confocal microscope equipped with PC-Apochromat 63x/1.2 NA water immersion and PC-Apochromat 20x/0.8 NA objective lenses was used for image acquisition. For detection of GFP and mCherry, 488-nm and 561-nm excitation wavelengths and BP 505-550 and LP 575 filters were used. During live-cell imaging, chromosomes labeled with H2B-mCherry were tracked with an EMBL-developed tracking macro (Rabut and Ellenberg, 2004) adapted to our microscope. We imaged 8 to 16 z-confocal sections (every 1.5 or $2.0 \mu \mathrm{m})$ at $5-10 \mathrm{~min}$ intervals for $12-14 \mathrm{hr}$.

\section{Chromosome spreads}

Chromosome spreads from mouse oocytes were prepared as previously described (Peters et alo, 1997; Hodges and Hunt, 2002). Briefly, zona pellucida was removed by treatment with $10 \mathrm{mg} / \mathrm{ml}$ Pronase (Sigma-Aldrich) at $37^{\circ} \mathrm{C}$ for $5-10 \mathrm{~min}$. Zona-free oocytes were transferred to hypotonic solution ( $50 \%$ FCS in deionized water) for $10 \mathrm{~min}$ at $37^{\circ} \mathrm{C}$. Oocytes were then fixed in paraformaldehyde solution ( $1 \%$ paraformaldehyde, $0.15 \%$ Triton X-100, 3 mM dithiothreitol, adjusted to $\mathrm{pH} 9.2$ with $\mathrm{NaOH}$ ) in 15-well multichamber glass slide (MP Biomedicals) overnight at room temperature in a humidified chamber. After drying, slides were washed twice in $0.4 \%$ Photoflo/ $\mathrm{H}_{2} \mathrm{O}$ (Kodak) and PBS for $5 \mathrm{~min}$, at room temperature. Slides were then processed for immunostaining. In this study, we used CREST (1:250; Davis Lab, Davis, CA, USA), rabbit anti-Sgol2 serum (1:50; gift from JL Barbero [Parra et al., 2009]), anti-phospho T521 Sgol2 (1:500, gift from Yoshi Watanabe [Tanno et al., 2010]), anti-MCAK (1:500, gift from Duane A Compton [Mack and Compton, 2001]), anti-phospho S24 Knl1 (1:1000, gift from lain Cheeseman [Welburn et al., 2010]), antiphospho S100 Dsn1 (1:500, gift from lain Cheeseman [Welburn et alo, 2010]), antibody detecting T288, T232 or T198 on Aurora A/B/C (1:50, Cell Signaling Technology, Danvers, MA, USA), anti-Aurora C (1:50, Bethly Laboratories, Montgomery, TX, USA), mouse anti-PP2A-C (1:200, BD Biosciences, Palo Alto, CA, USA), and anti-c-Myc (1:500, Millipore, Billerica, MA, USA).

\section{Immunofluorescence}

For analysis of K-fibers, oocytes were processed as previously described (Kitajima et al., 2011). Briefly, after either 2 or $6 \mathrm{hr}$ post-GVBD, oocytes were treated for $90 \mathrm{~s}$ with $\mathrm{Ca}^{2+}$ buffer (100 mM PIPES pH 7:0, $1 \mathrm{mM} \mathrm{MgCl} 2,0.1 \mathrm{mM} \mathrm{CaCl}, 0.1 \%$ Triton X-100) at $37^{\circ} \mathrm{C}$ and then fixed in $1 \%$ formaldehyde prepared in $\mathrm{Ca}^{2+}$ buffer for $30 \mathrm{~min}$ at room temperature. Following fixation, oocytes were washed and extracted overnight in PBT (PBS supplemented with $0.1 \%$ Triton X-100) at $4^{\circ} \mathrm{C}$. Cells were then blocked in $3 \%$ BSA with PBT (blocking solution) for $1 \mathrm{hr}$ at room temperature. For immuno-labeling oocytes were incubated overnight at $4^{\circ} \mathrm{C}$ with $\beta$-tubulin (1:250, Abcam, Cambridge, UK) and CREST (1:100, Davis Lab, Davis, CA, USA) 


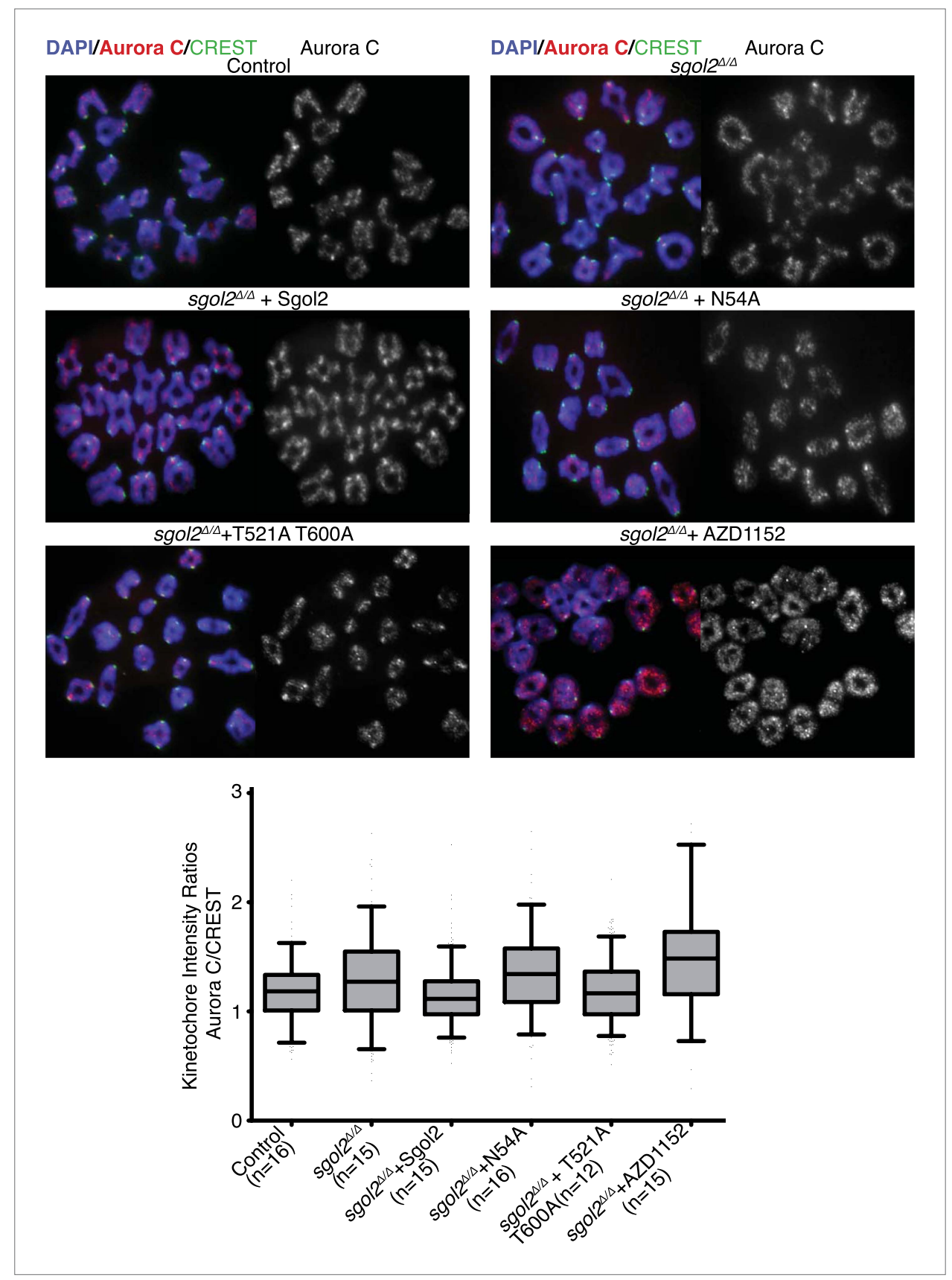

Figure 10. Sgol2 decreases Aurora C activity at the kinetochores without affecting its localization. Chromosome spreads were prepared from the indicated groups at $4 \mathrm{hr}$ post-GVBD. Slides were stained for DNA (DAPI, blue), Aurora C (red), and CREST (green). Fluorescence intensity ratios of Aurora C and CREST at kinetochores are displayed. Upper and lower bars indicate 95th and 5th percentiles, respectively. DOI: 10.7554/eLife.01133.025

antibodies, prepared in blocking solution. After four washes in PBT, oocytes were incubated for $1 \mathrm{hr}$ at room temperature with Alexa 488 and 568 conjugated secondary antibodies (1:500, Invitrogen, UK) prepared in blocking solution. Oocytes were briefly stained with Hoechst $33,342(20 \mathrm{mg} / \mathrm{ml})$ before confocal imaging.

\section{Protein expression and purification}

Recombinant MBP-Sgol2cc-His and MBP-Sgol2cc-Flag were purified from 11 of Escherichia coli BL21(DE3) RIPL (Stratagene, La Jolla, CA, USA) carrying corresponding expression cassettes in 


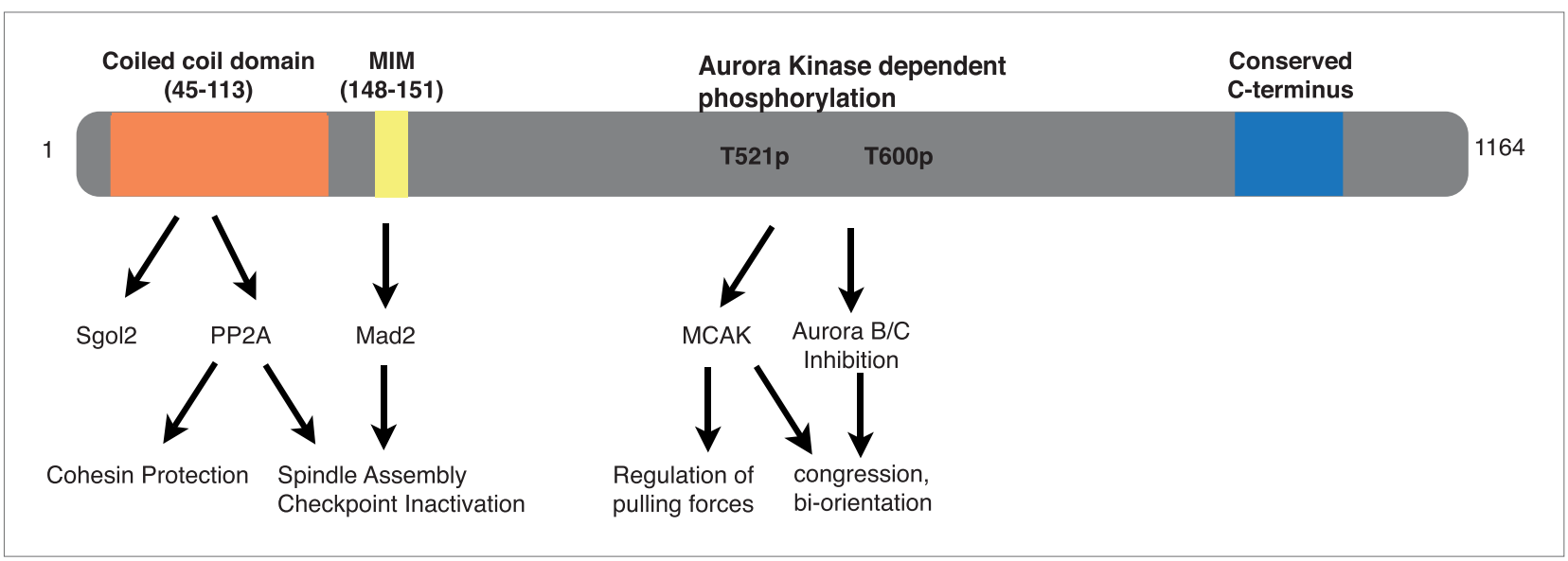

Figure 11. Graphical summary of sequence features, interactions and key functions of Sgol2 in mouse oocytes.

pMAL-c2x- (Flag-tagged subunit) and pET28b-based backbones (His-tagged subunit), grown at $37^{\circ} \mathrm{C}$ in 2xTY media supplemented with $25 \mu \mathrm{g} / \mathrm{ml}$ chloramphenicol and $100 \mu \mathrm{g} / \mathrm{ml}$ ampicillin for pMAL-c2x or $50 \mu \mathrm{g} / \mathrm{ml}$ kanamycin for pET28b. At $\mathrm{OD}_{600}=0.4, \mathrm{MBP}-\mathrm{Sgol} 2 \mathrm{cc}-\mathrm{His}$ or - Flag synthesis was induced at $30^{\circ} \mathrm{C}$ by the addition of $0.1 \mathrm{mM}$ IPTG. After $4 \mathrm{hr}$, the cells were harvested by centrifugation, frozen in dry ice, and stored at $-80^{\circ} \mathrm{C}$. The cell paste was re-suspended in $40 \mathrm{ml}$ of lysis buffer $(50 \mathrm{mM}$ sodium phosphate $\mathrm{pH} 7.0$, $500 \mathrm{mM} \mathrm{NaCl}, 10 \%$ glycerol, $0.02 \%$ Triton X-100) containing Roche complete EDTA free protease inhibitor, $1 \mathrm{mM}$ PMSF and, for the His-tagged subunit $5 \mathrm{mM}$. Each aliquot was sonicated four times for $30 \mathrm{~s}$ on ice. Insoluble material was removed by centrifugation at $25,000 \mathrm{rpm}$ for $1 \mathrm{hr}$ at $4^{\circ} \mathrm{C}$ in a Beckman Coulter Avanti-J26XP JLA8.1000 rotor. The supernatant was incubated for $2 \mathrm{hr}$ at $4^{\circ} \mathrm{C}$ on a $5 \mathrm{ml}$ Talon column (BD Biosciences, Palo Alto, CA, USA) or ANTI-FLAG M2 Affinity Gel (Sigma A2220). The M2 Flag beads were washed four times with $50 \mathrm{ml}$ of lysis buffer and the Talon beads were washed two times with $50 \mathrm{ml}$ of lysis buffer containing $20 \mathrm{mM}$ imidazole and two times with $50 \mathrm{ml}$ of lysis buffer containing $45 \mathrm{mM}$ imidazole. MBP-Sgol2cc-Flag was eluted with four times $1 \mathrm{ml}$ of lysis buffer containing $3 \times$ FLAG peptide $(150 \mathrm{ng} / \mu \mathrm{l})$. MBP-Sgol2cc-His was eluted with $10 \mathrm{ml}$ of lysis buffer containing $1 \mathrm{M}$ of imidazole. The concentration of MBP-Sgol2cc-His and MBP-Sgol2cc-Flag were determined by nanodrop against the lysis solution.

\section{PP2A-Sgol2 In vitro binding assay}

For all binding assays, highly-purified PP2A subunits (purified as previously described [Cho and Xu, 2007]) and MBP-Sgol2cc-Flag were used. To test the binding of PP2A to mutant shugoshin, $20 \mu \mathrm{g}$ of GST-tagged PP2A A subunit were incubated with excess amounts of PP2A B56- and C- subunit and MBP-tagged shugoshin for $1 \mathrm{hr}$ on ice. Subsequently, samples were incubated with $40 \mu \mathrm{l}$ of glutathione beads (GE Healthcare) for $20 \mathrm{~min}$. Beads were then washed $4 \times$ with $1 \mathrm{ml}$ each of $1 \times$ PBS, $0.1 \%$ Triton X-100, $1 \mathrm{mM}$ dithiothreitol, boiled in reducing SDS loading buffer, and subjected to SDS-PAGE analysis followed by Coomassie blue staining.

\section{Co-immunoprecipitation of Sgol2 coiled coil domains}

His and Flag-tagged $\mathrm{mSgol} 2 \mathrm{cc}$ from wild type and 3A were co-expressed in E.coli BL21(DE3)RIPL for $4 \mathrm{hr}$ at $30^{\circ} \mathrm{C}$ with $0.1 \mathrm{mM}$ of IPTG. Cells were spun at $25,000 \mathrm{rpm}$ for $1 \mathrm{hr}$ at $4^{\circ} \mathrm{C}$ in a Beckman Coulter AvantiJ26XP JLA8.1000 rotor. The pellet was re-suspended into lysis buffer ( $50 \mathrm{mM}$ sodium phosphate $\mathrm{pH}=7$; $250 \mathrm{mM} \mathrm{NaCl}$; $10 \%$ glycerol, $0.02 \%$ Triton X-100; protease inhibitor). $10 \mathrm{mg}$ of extract were incubated with $100 \mu$ of Talon beads for $2 \mathrm{hr}$ at $4^{\circ} \mathrm{C}$. The beads were wash five times with the lysis buffer containing $23 \mathrm{mM}$ of imidazole. MBP-Sgol2cc-His was eluted with $10 \mathrm{ml}$ of lysis buffer containing $1 \mathrm{M}$ of imidazole. Affinity purified material and input were resolved by SDS-PAGE. The abundance of His and Flag-tagged MBPSgol2cc were analyzed by western blot using anti-Flag $(1: 10,000)$ and anti-His $(1: 10,000)$ antibodies.

\section{Acknowledgements}

We would like to thank members of the Nasmyth lab, Bela Novak, and Elwy Okaz for discussions and advise, and Melina Schuh for critical reading of the manuscript. We thank Lysie Champion and 
Kikue Tachibana-Konwalski for the pRNA-CENPB-eGFP vector, and Jan Ellenberg for sharing the pGEMHE-EB3-eGFP construct. We are grateful to lain Cheeseman, Arshad Desai, Duane A Compton, JL Barbero, Jason Swedlow, Yoshi Watanabe, and Linda Wordeman for providing antibodies. We would like to thank Elena Llano for providing the Sgol2 targeted embryos. We would also like to thank Chris Preece and Ben Davies for valuable microinjection training, Michael Orth and Bulent Cetin for sharing their unpublished findings, and animal care staff at the BSB Facility, Dept. of Biomedical Services for technical assistance. AR was supported by a PhD fellowship from the Boehringer Ingelheim Fonds. BM and OS were supported by a grant of the Deutsche Forschungsgemeinschaft (STE997/3-2 within the priority program SPP1384). AP was supported by SAF2011-25252. This study was funded by the European Community's Seventh Framework MitoSys/241548, Medical Research Council and Wellcome Trust.

\section{Additional information}

Funding

\begin{tabular}{lll} 
Funder & $\begin{array}{l}\text { Grant reference } \\
\text { number }\end{array}$ & Author \\
\hline Wellcome Trust & $019859 / Z / 10 / Z$ & Kim Nasmyth \\
\hline Medical Research Council & 84673 & Kim Nasmyth \\
\hline Boehringer Ingelheim Fonds & & Ahmed Rattani \\
\hline Deutsche Forschungsgemeinschaft & $\begin{array}{l}\text { STE997/3-2 within the } \\
\text { priority program SPP1384 }\end{array}$ & Olaf Stemmann \\
\hline Ministerio de Ciencia e Innovacion & SAF2011-25252 & Alberto Pendas \\
\hline European Community's Seventh & MitoSys/241548 & Kim Nasmyth \\
Framework Programme & &
\end{tabular}

The funders had no role in study design, data collection and interpretation, or the decision to submit the work for publication.

Author contributions

AR, Conception and design, Acquisition of data, Analysis and interpretation of data, Drafting or revising the article; MW, MP, WH, SM, JG, Acquisition of data, Analysis and interpretation of data; $\mathrm{BM}$, Acquisition of data, Analysis and interpretation of data, Drafting or revising the article; WX, Analysis and interpretation of data, Contributed unpublished essential data or reagents; OS, Drafting or revising the article, Contributed unpublished essential data or reagents; AP, KN, Conception and design, Drafting or revising the article

Ethics

Animal experimentation: Animal studies were carried out under the UK Home Office ASPA project licence 30/2824.

\section{References}

Akiyoshi B, Sarangapani KK, Powers AF, Nelson CR, Reichow SL, Arellano-Santoyo H, et al. 2010. Tension directly stabilizes reconstituted kinetochore-microtubule attachments. Nature 468:576-9. doi: 10.1038/nature09594.

Andrews PD, Ovechkina Y, Morrice N, Wagenbach M, Duncan K, Wordeman L, et al. 2004. Aurora B regulates MCAK at the mitotic centromere. Dev Cell 6:253-68. doi: 10.1016/S1534-5807(04)00025-5.

Cho US, Xu W. 2007. Crystal structure of a protein phosphatase 2A heterotrimeric holoenzyme. Nature 445:53-7. doi: $10.1038 /$ nature05351.

Hassold T, Hunt P. 2001. To err (meiotically) is human: the genesis of human aneuploidy. Nat Rev Genet 2:280-91. doi: $10.1038 / 35066065$.

Hodges CA, Hunt PA. 2002. Simultaneous analysis of chromosomes and chromosome-associated proteins in mammalian oocytes and embryos. Chromosoma 111:165-9. doi: 10.1007/s00412-002-0195-3.

Holland AJ, Bottger F, Stemmann O, Taylor SS. 2007. Protein phosphatase 2A and separase form a complex regulated by separase autocleavage. J Biol Chem 282:24623-32. doi: 10.1074/jbc. M702545200.

Huang H, Feng J, Famulski J, Rattner JB, Liu ST, Kao GD, et al. 2007. Tripin/hSgo2 recruits MCAK to the inner centromere to correct defective kinetochore attachments. J Cell Biol 177:413-24. doi: 10.1083/jcb.200701122. Ishiguro T, Tanaka K, Sakuno T, Watanabe Y. 2010. Shugoshin-PP2A counteracts casein-kinase-1-dependent cleavage of Rec8 by separase. Nat Cell Biol 12:500-6. doi: 10.1038/ncb2052. 
Katis VL, Lipp JJ, Imre R, Bogdanova A, Okaz E, Habermann B, et al. 2010. Rec8 phosphorylation by casein kinase 1 and Cdc7-Dbf4 kinase regulates cohesin cleavage by separase during meiosis. Dev Cell 18:397-409. doi: 10.1016/j.devcel.2010.01.014.

Kitajima TS, Ohsugi M, Ellenberg J. 2011. Complete kinetochore tracking reveals error-prone homologous chromosome biorientation in mammalian oocytes. Cell 146:568-81. doi: 10.1016/j.cell.2011.07.031.

Kudo NR, Wassmann K, Anger M, Schuh M, Wirth KG, Xu H, et al. 2006. Resolution of chiasmata in oocytes requires separase-mediated proteolysis. Cell 126:135-46. doi: 10.1016/j.cell.2006.05.033.

Lampson MA, Cheeseman IM. 2011. Sensing centromere tension: Aurora B and the regulation of kinetochore function. Trends Cell Biol 21:133-40. doi: 10.1016/j.tcb.2010.10.007.

Lee J, Kitajima TS, Tanno Y, Yoshida K, Morita T, Miyano T, et al. 2008. Unified mode of centromeric protection by shugoshin in mammalian oocytes and somatic cells. Nat Cell Biol 10:42-52. doi: 10.1038/ncb1667.

Liu D, Vader G, Vromans MJ, Lampson MA, Lens SM. 2009. Sensing chromosome bi-orientation by spatial separation of aurora B kinase from kinetochore substrates. Science 323:1350-3. doi: 10.1126/science.1167000.

Liu H, Rankin S, Yu H. 2013. Phosphorylation-enabled binding of SGO1-PP2A to cohesin protects sororin and centromeric cohesion during mitosis. Nat Cell Biol 15:40-9. doi: 10.1038/ncb2637.

Llano E, Gomez R, Gutierrez-Caballero C, Herran Y, Sanchez-Martin M, Vazquez-Quinones L, et al. 2008. Shugoshin-2 is essential for the completion of meiosis but not for mitotic cell division in mice. Genes Dev 22:2400-13. doi: 10.1101/gad.475308.

Mack GJ, Compton DA. 2001. Analysis of mitotic microtubule-associated proteins using mass spectrometry identifies astrin, a spindle-associated protein. Proc Natl Acad Sci USA 98:14434-9. doi: 10.1073/ pnas.261371298.

McGuinness BE, Hirota T, Kudo NR, Peters JM, Nasmyth K. 2005. Shugoshin prevents dissociation of cohesin from centromeres during mitosis in vertebrate cells. PLOS Bio/ 3:e86. doi: 10.1371/journal.pbio.0030086.

Orth M, Mayer B, Rehm K, Rothweiler U, Heidmann D, Holak TA, et al. 2011. Shugoshin is a Mad1/Cdc20-like interactor of Mad2. EMBO J 30:2868-80. doi: 10.1038/emboj.2011.187.

Parra MT, Gomez R, Viera A, Llano E, Pendas AM, Rufas JS, et al. 2009. Sequential assembly of centromeric proteins in male mouse meiosis. PLOS Genet 5:e1000417. doi: 10.1371/journal.pgen.1000417.

Peters AH, Plug AW, van Vugt MJ, de Boer P. 1997. A drying-down technique for the spreading of mammalian meiocytes from the male and female germline. Chromosome Res 5:66-8. doi: 10.1023/A:1018445520117.

Rabut G, Ellenberg J. 2004. Automatic real-time three-dimensional cell tracking by fluorescence microscopy. J Microsc 216:131-7. doi: 10.1111/j.0022-2720.2004.01404.x.

Rivera T, Ghenoiu C, Rodriguez-Corsino M, Mochida S, Funabiki H, Losada A. 2012. Xenopus Shugoshin 2 regulates the spindle assembly pathway mediated by the chromosomal passenger complex. EMBO J 31:1467-79. doi: 10.1038/emboj.2012.4.

Schuh M, Ellenberg J. 2007. Self-organization of MTOCs replaces centrosome function during acentrosomal spindle assembly in live mouse oocytes. Cell 130:484-98. doi: 10.1016/j.cell.2007.06.025.

Tachibana-Konwalski K, Godwin J, van der Weyden L, Champion L, Kudo NR, Adams DJ, et al. 2010. Rec8-containing cohesin maintains bivalents without turnover during the growing phase of mouse oocytes. Genes Dev 24:2505-16. doi: 10.1101/gad.605910.

Tanno Y, Kitajima TS, Honda T, Ando Y, Ishiguro K, Watanabe Y. 2010. Phosphorylation of mammalian Sgo2 by Aurora B recruits PP2A and MCAK to centromeres. Genes Dev 24:2169-79. doi: 10.1101/gad.1945310.

Tsukahara T, Tanno Y, Watanabe Y. 2010. Phosphorylation of the CPC by Cdk1 promotes chromosome bi-orientation. Nature 467:719-23. doi: 10.1038/nature09390.

Welburn JP, Vleugel M, Liu D, Yates JR, Lampson MA, Fukagawa T, et al. 2010. Aurora B phosphorylates spatially distinct targets to differentially regulate the kinetochore-microtubule interface. Mol Cell 38:383-92. doi: 10.1016/j.molcel.2010.02.034.

Xu Z, Cetin B, Anger M, Cho US, Helmhart W, Nasmyth K, et al. 2009. Structure and function of the PP2A-shugoshin interaction. Mol Cell 35:426-41. doi: 10.1016/j.molcel.2009.06.031.

Yamagishi Y, Honda T, Tanno Y, Watanabe Y. 2010. Two histone marks establish the inner centromere and chromosome bi-orientation. Science 330:239-43. doi: 10.1126/science.1194498. 